Article

\title{
Further Results of the TTT Transform Ordering of Order $n$
}

\author{
Lei Yan ${ }^{1}$ (D), Diantong Kang ${ }^{2}$ and Haiyan Wang ${ }^{1, *(D)}$ \\ 1 Business School, Zhejiang Wanli University, Ningbo 315100, China; yanlei@zwu.edu.cn \\ 2 School of Mathematics and Statistics, Hexi University, Zhangye 734000, China; dtkang@hxu.edu.cn \\ * Correspondence: wanghaiyan@zwu.edu.cn
}

\section{check for}

updates

Citation: Yan, L.; Kang, D.T.; Wang, H.Y. Further Results of the TTT Transform Ordering of Order $n$. Symmetry 2021, 13, 1960. https:// doi.org/10.3390/sym13101960

Academic Editor: Michael Greenacre

Received: 26 August 2021

Accepted: 14 October 2021

Published: 18 October 2021

Publisher's Note: MDPI stays neutral with regard to jurisdictional claims in published maps and institutional affiliations.

\begin{abstract}
To compare the variability of two random variables, we can use a partial order relation defined on a distribution class, which contains the anti-symmetry. Recently, Nair et al. studied the properties of total time on test (TTT) transforms of order $n$ and examined their applications in reliability analysis. Based on the TTT transform functions of order $n$, they proposed a new stochastic order, the TTT transform ordering of order $n$ (TTT- $n$ ), and discussed the implications of order TTT- $n$. The aim of the present study is to consider the closure and reversed closure of the TTT-n ordering. We examine some characterizations of the TTT- $n$ ordering, and obtain the closure and reversed closure properties of this new stochastic order under several reliability operations. Preservation results of this order in several stochastic models are investigated. The closure and reversed closure properties of the TTT- $n$ ordering for coherent systems with dependent and identically distributed components are also obtained.
\end{abstract}

Keywords: TTT transform of order $n$; TTT transform ordering of order $n$; closure property; reversed closure property; mixture proportional reversed hazard rate model; coherent system

\section{Introduction}

"Symmetry" is usually used to refer to an object that is invariant under some transformations; including translation, reflection, etc. (for example, Zee [1]). The opposite of symmetry is ant-symmetry, which refers to the absence or a violation of symmetry. As we known, the deterministic, stochastic and fractional mathematical models are widely used in many different research fields. The research objects of these three models are distinct, and their research methods and results are also different. In these directions, interested readers can refer to, for instance, Din and Li [2], Din et al. [3], Kosec et al. [4], Din et al. [5], and the references therein. In statistics, symmetry also manifests as symmetric probability distributions, and as skewness - the anti-symmetry of distributions (see Petitjean [6]). Tahmasebi et al. [7] study the symmetry property of independent random variables with a joint distribution function. Especially in stochastic models, it is of interest to compare the variability of random variables, and a nice way is by a partial order relation defined on a distribution class. Sometimes, we need to infer the properties of individual (or a unit) from the properties of a population (or a system), then we say this order has closed properly, whereas, when we do such things from the opposite direction in other times, then reversely we say this order has reversed closure. If a stochastic order is closed with respect to some system, but not reversely closed; or a stochastic order is reversely closed with respect to some system, rather than closed, we can regard this stochastic order to have a kind of anti-symmetry. Whether a stochastic order has the closure or reversed closure is thus worth studying. This symmetry or anti-symmetry is conducive to uncertainty management.

Let $X$ be a non-negative and absolutely continuous random variable with distribution function $F_{X}$ and survival function $\bar{F}_{X}=1-F_{X}$, and density function $f_{X}$, respectively. The quantile function of $F_{X}$ is defined by

$$
Q_{X}(p)=F_{X}^{-1}(p)=\inf \{x \mid F(x) \geq p\}, \quad \text { for all } p \in(0,1) .
$$


The concept of total time on test (TTT) transforms is of significant importance for its applications in different study fields such as reliability theory and economics. It was proposed by Barlow et al. [8], and subsequently developed by Barlow and Campo [9], Klefsjö [10], Bartoszewicz [11-14], Pham and Turkkan [15], Li and Shaked [16], Nanda and Shaked [17], and among others. The TTT transform function of X is defined as (see Shaked and Shanthikumar [18])

$$
T_{X}(p)=\int_{0}^{F_{X}^{-1}(p)} \bar{F}_{X}(x) \mathrm{d} x, \quad \text { for all } p \in(0,1) .
$$

Note that $T_{X}(1)=\mathbb{E}[X]$, where the expectation $\mathbb{E}[X]=\mu_{X}$ can be finite or infinite.

Barlow and Campo [9] found that the TTT function $T_{X}$ in (2) is increasing in $(0,1)$, and hence it can be viewed as the inverse of a distribution function $H_{X}$ of a random variable with support in $\left(0, T_{X}(1)\right)=\left(0, \mu_{X}\right)$, where the mean $\mu_{X}$ may be finite or infinite. $\mathrm{Li}$ and Shaked [16] investigated this distribution function. Furthermore, based on the TTT transform function, they defined the observed TTT random variable, written as $X_{\mathrm{ttt}}$, by the distribution function with support in $\left(0, \mu_{X}\right)$ :

$$
H_{X}(y)=\mathbb{P}\left\{X_{\mathrm{ttt}} \leq y\right\}=T_{X}^{-1}(y), \quad y \in\left(0, \mu_{X}\right) ;
$$

the corresponding density function $h_{X}=H_{X}^{\prime}$ is given by

$$
h_{X}(y)=\frac{1}{t_{X}\left(T_{X}^{-1}(y)\right)}, \quad y \in\left(0, \mu_{X}\right),
$$

where $t_{X}(p)=\frac{\mathrm{d}}{\mathrm{d} p} T_{X}(p)$ is the TTT density of $X$.

They also showed that

$$
X_{\mathrm{ttt}}=T_{X}\left(F_{X}(X)\right)=\int_{0}^{X} \bar{F}_{X}(x) \mathrm{d} x
$$

it literally measures the observed total time on test when $X$ is observed (see Franco-Pereira and Shaked [19]).

The random variable $X_{\mathrm{ttt}}$ has some useful applications in reliability theory; see Li and Shaked [16] and Franco-Pereira and Shaked [19].

By using the TTT transform functions, Kochar et al. [20] established the following TTT transform ordering and gave this stochastic order a careful study.

Let $X$ and $Y$ be two non-negative random variables with distribution functions $F_{X}, G_{Y}$, survival functions $\bar{F}_{X} \equiv 1-F_{X}, \bar{G}_{Y} \equiv 1-G_{Y}$, quantile functions $F_{X}^{-1}, G_{Y}^{-1}$, respectively. $X$ is said to be smaller than $Y$ in the TTT transform ordering (denoted by $X \leq_{\mathrm{ttt}} Y$ ) if

$$
\int_{0}^{F_{X}^{-1}(p)} \bar{F}_{X}(x) \mathrm{d} x \leq \int_{0}^{G_{Y}^{-1}(p)} \bar{G}_{Y}(x) \mathrm{d} x, \quad \text { for all } p \in(0,1) .
$$

If $\bar{F}_{X}(x) \leq \bar{G}_{Y}(x), x \in \Re$ (equivalently, $F_{X}^{-1}(p) \leq G_{Y}^{-1}(p)$ for all $p \in(0,1)$ ), then $X$ is said to be smaller than $Y$ in the usual stochastic order (denoted by $X \leq_{\text {st }} Y$ or $F_{X} \leq_{\text {st }} G_{Y}$ ); this order is studied, for example, by Shaked and Shanthikumar [18]. Jewitt [21] introduced the following order. If

$$
\int_{-\infty}^{F_{X}^{-1}(p)} F_{X}(x) \mathrm{d} x \leq \int_{-\infty}^{G_{Y}^{-1}(p)} G_{Y}(x) \mathrm{d} x, \quad p \in(0,1),
$$

provided that the integrals are finite, then $X$ is said to be smaller than $Y$ in the location independent riskier order (denoted by $X \leq_{\operatorname{lir}} Y$ ). Fagiuoli et al. [22] and Kochar et al. [20] further studied this order. 
It is well-known that if $X$ and $Y$ are non-negative with common left endpoint 0 of their supports, then

$$
X \leq_{\text {lir }} Y \Longrightarrow X \leq_{\text {st }} Y \Longrightarrow X \leq_{\mathrm{ttt}} Y .
$$

See Shaked and Shanthikumar [18].

A generalization of the TTT ordering is described next. This generalization contains as special cases the orders $\leq_{\mathrm{st}}, \leq_{\text {lir }}$, and $\leq_{\mathrm{ttt}}$.

$\mathrm{Li}$ and Shaked [16] introduced and studied a family of univariate stochastic orders parameterized by a function $h$. Let $\mathfrak{H}$ denote the set of all functions $h$ such that $h(u)>0$ for $u \in(0,1)$, and $h(u)=0$ for $u \notin[0,1]$. For $h \in \mathfrak{H}$, denote by

$$
T_{X}(p ; h)=\int_{0}^{F_{X}^{-1}(p)} h\left[F_{X}(x)\right] \mathrm{d} x \quad \text { and } \quad T_{Y}(p ; h)=\int_{0}^{G_{Y}^{-1}(p)} h\left[G_{Y}(x)\right] \mathrm{d} x,
$$

for all $p \in(0,1) . T_{X}(p ; h)$ and $T_{Y}(p ; h)$ are called the generalized TTT (GTTT) transforms of $X$ and $Y$ with respect to $h$, respectively. Based on the GTTT transform functions, they defined the following new stochastic order. Let $\mathrm{h}$ be a function as described in $\mathrm{Li}$ and Shaked [16] as above.

Let $X$ and $Y$ be two non-negative random variables. Let $h$ be a function as described in Li and Shaked [16]. $X$ is said to be smaller than $Y$ in the generalized TTT (GTTT) ordering with respect to $h$ (denoted by $X \leq_{\mathrm{ttt}}^{(h)} Y$ or $F_{X} \leq_{\mathrm{ttt}}^{(h)} G_{Y}$ ) if

$$
\int_{0}^{F_{X}^{-1}(p)} h\left[F_{X}(x)\right] \mathrm{d} x \leq \int_{0}^{G_{Y}^{-1}(p)} h\left[G_{Y}(x)\right] \mathrm{d} x, \quad \text { for all } p \in(0,1) .
$$

They showed that if $h$ is a constant function on $[0,1]$; that is, $h(u)=c, u \in[0,1]$, for some $c>0$, and $h(u)=0$ otherwise. Then, the order $\leq_{\mathrm{ttt}}^{(h)}$ becomes as the order $\leq_{\mathrm{st}}$. If $h(u)=u, u \in[0,1]$, and $h(u)=0$ otherwise. Then, the order $\leq_{\mathrm{ttt}}^{(h)}$ becomes as the order $\leq_{\text {lir. }}$ If $h(u)=1-u, u \in[0,1]$, and $h(u)=0$ otherwise. Then, the order $\leq_{\mathrm{ttt}}^{(h)}$ becomes as the order $\leq_{\mathrm{ttt}}$. They also studied some properties of this family, and gave some applications of it in actuarial science, reliability theory, and statistics. Shaked and Shanthikumar [18] described a relationship among the orders $\leq_{\mathrm{ttt}}^{(h)} Y$ for different $h$ 's. For more details about the GTTT ordering, we also refer to Shaked and Shanthikumar [18].

Nair et al. [23] studied the TTT transform functions of order $n$ (see Section 2 below), based on the TTT transform functions of order $n$, they introduced the TTT transform ordering of order $n$ (TTT- $n$ ordering, for short), and studied the properties of this new order. They also examined the implications between the TTT- $n$ ordering and some other stochastic orders often used in reliability analysis. The aging properties of the baseline distribution was compared with those of transformed distributions.

Recently, Bartoszewicz and Benduch [14] studied some properties of the GTTT transforms. They made stochastic comparisons of GTTT transforms in several commonly used stochastic orders. They defined invariance properties and distances of some stochastic orders by using the GTTT transforms. Iterations of the GTTT transforms are also studied, and their relations with exponential mixtures of gamma distributions are established. Nair and Sankaran [23] presented some new applications of the total time on test transforms. They presented four applications of TTT in reliability theory. They characterized aging criteria such as IFRA and NBU in terms of TTT. They utilized an iterated version to construct bathtub shaped hazard quantile functions and corresponding lifetime models. Further, an index was developed for numerically measuring the extent of IFR-ness of a life distribution. They also demonstrated how the distributional properties such as kurtosis and skewness can be derived from the TTT.

More recently, Franco-Pereira and Shaked [19] studied the TTT transform and the decreasing percentile residual life aging notion. On the basis of the work of Nair and Sankaran [23], they added two characterizations of the decreasing percentile residual life of order $\alpha(\operatorname{DPRL}(\alpha))$ aging notion in terms of the TTT function, and in terms of the observed 
TTT when $X$ is observed. Vineshkumar et al. [24] studied the TTT and TTT- $n$ orderings by using quantile-based reliability functions. They developed new stochastic orders using the quantile-based reliability measures like the hazard quantile function and the mean residual quantile function. They also established relationships among the proposed orders and certain existing orders. Various properties of the orders are also studied.

The following lemma taken from Barlow and Proschan ([25], p. 120) is useful in the sequel.

Lemma 1. Let $W$ be a measure on the interval $(a, b)$, not necessarily non-negative, where $-\infty \leq$ $a<b \leq+\infty$. Let $h$ be a non-negative function defined on $(a, b)$. If $\int_{a}^{t} d W(x) \geq 0$ for all $t \in(a, b)$ and if $h$ is decreasing, then

$$
\int_{a}^{b} h(x) d W(x) \geq 0
$$

In this paper, we focus our interest on the further properties of the TTT- $n$ ordering, especially the closure and reversed closure properties of this order. The organization of the paper is as follows. In Section 2, we explore the characterizations of the TTT- $n$ ordering. In Section 3, we investigate the closure and reversed closure properties of the TTT ordering. As applications of a main result Theorem 1, in Section 4, we examine the preservation of the TTT- $n$ ordering in several stochastic models. In Section 5 , we obtain the closure and reversed closure properties of this order for coherent systems. Section 6 is the conclusion of this research.

The highlights of our research are: (1) the TTT- $n$ ordering is closed respect to a series system and a random series system, respectively; (2) the TTT- $n$ ordering is reversely closed respect to a parallel system and reversely closed respect to a random parallel system, respectively; (3) the TTT- $n$ ordering is closed under a non-negative, increasing and concave transform; (4) the TTT- $n$ ordering is reversely closed under a non-negative and increasing convex transform; (5) the TTT- $n$ ordering is closed and reversely closed, respectively, under some appropriate conditions in several stochastic models. (6) We summarize the research results of this article, and obtained 17 results concerning anti-symmetry.

In the paper, the term increasing stands for monotone non-decreasing and decreasing stands for monotone non-increasing. Assume that all random variables involved are absolutely continuous and non-negative, and that all integrals appeared are finite and all ratios are well defined whenever written.

\section{Characterizations of the TTT Transform Ordering of Order $n$}

Let $X$ and $Y$ be two absolutely continuous and non-negative random variables with distribution functions $F_{X}$ and $G_{Y}$, survival functions $\bar{F}_{X}=1-F_{X}$ and $\bar{G}_{Y}=1-G_{Y}$, density functions $f_{X}$ and $g_{Y}$, and quantile functions $F_{X}^{-1}$ and $G_{Y}^{-1}$ of $F_{X}$ and $G_{Y}$, respectively. $X$ and $Y$ have 0 as the common left endpoint of their supports.

Recently, Nair et al. [26] studied the TTT transforms of order $n$, and based on the TTT transforms of order $n$, they introduced and examined the TTT transform ordering of order $n$. They exploited the implications between the TTT transform ordering of order $n$ and some other stochastic orders often used in reliability analysis. They recursively defined the TTT transforms of order $n$ of a non-negative continuous random variable $X$ given by

$$
T_{n}^{X}(p)=\int_{0}^{p}(1-u) t_{n-1}^{X}(u) \mathrm{d} u, \quad n=1,2, \ldots,
$$

with $T_{0}^{X}(p)=Q_{X}(p)$ and

$$
t_{n}^{X}(p)=\frac{\mathrm{d}}{\mathrm{d} p} T_{n}^{X}(p), \quad t_{0}^{X}(p)=\frac{\mathrm{d}}{\mathrm{d} p} T_{0}^{X}(p)=\frac{\mathrm{d}}{\mathrm{d} p} Q_{X}(p)=q_{X}(p)=\frac{1}{f_{X}\left[F_{X}(p)\right]},
$$

provided that $\mu_{n-1}^{X}=\int_{0}^{1}(1-u) t_{n-1}^{X}(u) \mathrm{d} u<\infty$. 
They denoted by $X_{n}$ the random variable with quantile function $T_{n}^{X}(p)$ and mean $\mu_{n}^{X}$. By differentiating (5), they showed that

$$
t_{n}^{X}(p)=(1-p) t_{n-1}^{X}(p)
$$

and

$$
t_{n}^{X}(p)=(1-p)^{n} t_{0}^{X}(p)=(1-p)^{n} q_{X}(p) .
$$

From (5) and (6), we have

$$
T_{n}^{X}(p)=\int_{0}^{p}(1-u)^{n} q_{X}(u) \mathrm{d} u, \quad n=1,2, \ldots
$$

Letting $u=F_{X}(x)$ in the above integral, we thus get

$$
T_{n}^{X}(p)=\int_{0}^{F_{X}^{-1}(p)}\left[\bar{F}_{X}(x)\right]^{n} \mathrm{~d} x, \quad n=1,2, \ldots
$$

Definition 1 (Nair et al. [26]). Let $X$ and $Y$ be two non-negative random variable, $X$ is said to be smaller than $Y$ in the TTT transform of order $n$, written as $X \leq_{t t t-n} Y$ (or, equivalently, $\left.X_{n} \leq_{t t t} Y_{n}\right)$, if $T_{n+1}^{X}(p) \leq T_{n+1}^{Y}(p)$ for all $p \in(0,1)$, where $T_{n}^{X}(p)$ and $T_{n}^{Y}(p)$ denote the TTT transforms of order $n$ of $X$ and $Y$, respectively.

Below, we give a necessary and sufficient condition of the TTT ordering of order $n$, which will play a key role in the proofs of the results in the whole paper.

Theorem 1. $X \leq_{t t t-n} Y$ if and only if

$$
\int_{0}^{t}\left[\bar{F}_{X}(x)\right]^{n+1}\left[\frac{f_{X}(x)}{g_{Y}\left[G_{Y}^{-1}\left(F_{X}(x)\right)\right]}-1\right] d x \geq 0, \text { for all } t \geq 0 .
$$

Proof. From Definition 1 and (8) we have $X \leq_{\mathrm{ttt}-n} Y$ if and only if

$$
\int_{0}^{F_{X}^{-1}(p)}\left[\bar{F}_{X}(x)\right]^{n+1} \mathrm{~d} x \leq \int_{0}^{G_{Y}^{-1}(p)}\left[\bar{G}_{Y}(x)\right]^{n+1} \mathrm{~d} x, \quad \text { for all } p \in[0,1] .
$$

Letting $\bar{G}_{Y}(x)=\bar{F}_{X}(y)$ in the right-hand side of inequality (10) that is, $x=G_{Y}^{-1}\left[F_{X}(y)\right]$, and $\mathrm{d} x=\left(f_{X}(y) / g_{Y}\left[G_{Y}^{-1}\left(F_{X}(y)\right)\right]\right) \mathrm{d} y$, we have, for all $p \in[0,1]$,

$$
\begin{gathered}
\int_{0}^{G_{Y}^{-1}(p)}\left[\bar{G}_{Y}(x)\right]^{n+1} \mathrm{~d} x=\int_{0}^{F_{X}^{-1}(p)}\left[\bar{F}_{X}(y)\right]^{n+1} \frac{f_{X}(y)}{g_{Y}\left[G_{Y}^{-1}\left(F_{X}(y)\right)\right]} \mathrm{d} y \\
=\int_{0}^{F_{X}^{-1}(p)}\left[\bar{F}_{X}(x)\right]^{n+1} \frac{f_{X}(x)}{g_{Y}\left[G_{Y}^{-1}\left(F_{X}(x)\right)\right]} \mathrm{d} x .
\end{gathered}
$$

Thus, from inequality (10), we have

$$
\int_{0}^{F_{X}^{-1}(p)}\left[\bar{F}_{X}(x)\right]^{n+1}\left[\frac{f_{X}(x)}{g_{Y}\left[G_{Y}^{-1}\left(F_{X}(x)\right)\right]}-1\right] \mathrm{d} x \geq 0, \quad \text { for all } p \in[0,1] .
$$


Now, letting $F_{X}^{-1}(p)=t$, we find that

$$
\int_{0}^{t}\left[\bar{F}_{X}(x)\right]^{n+1}\left[\frac{f_{X}(x)}{g_{Y}\left[G_{Y}^{-1}\left(F_{X}(x)\right)\right]}-1\right] \mathrm{d} x \geq 0, \quad \text { for all } t \geq 0 .
$$

This completes the proof.

On using $Q_{X}^{\prime}(u)=q_{X}(u)=\left[f_{X}\left(F_{X}^{-1}(x)\right)\right]^{-1}, Q_{Y}^{\prime}(u)=q_{Y}(u)=\left[g_{Y}\left(G_{Y}^{-1}(x)\right)\right]^{-1}$ (also see Nanda et al. [27], Sunoj and Sankaran [28], Sunoj et al. [29]), as a direct consequence of Theorem 1, we get the following result immediately.

Corollary 1. $X \leq_{t t t-n} Y$ if and only if

$$
\int_{0}^{p}(1-u)^{n+1}\left[q_{Y}(u)-q_{X}(u)\right] d u \geq 0, \quad \text { for all } p \in[0,1] .
$$

Let $X$ be a non-negative continuous random variable with distribution function $F_{X}$ and mean $\mu_{X}<\infty$. Li and Shaked [30] defined the observed total time on test and the observed excess wealth random variables when $X$ is observed, and denoted by $X_{\mathrm{ttt}}$ and $X_{\text {ew }}$, respectively. They showed that

$$
X_{\mathrm{ttt}}=T_{X}\left(F_{X}(X)\right)={ }_{\mathrm{st}} T_{X}(U)=\int_{0}^{X} \bar{F}_{X}(x) \mathrm{d} x
$$

and

$$
X_{\mathrm{ew}}=W_{X}\left(F_{X}(X)\right)={ }_{\mathrm{st}} W_{X}(U)=\int_{X}^{\infty} \bar{F}_{X}(x) \mathrm{d} x,
$$

where $U$ is a uniform $(0,1)$ random variable, and $=_{\text {st }}$ denotes equality in distribution. They also gave that

$$
\begin{gathered}
X_{\mathrm{ew}}=\mu_{X}-X_{\mathrm{ttt}}, \quad \text { and } \\
\mathbb{E}\left[X_{\mathrm{ttt}}\right]=\int_{0}^{\infty} \bar{F}_{X}^{2}(x) \mathrm{d} x, \quad \mathbb{E}\left[X_{\mathrm{ew}}\right]=\int_{0}^{\infty} F_{X}(x) \bar{F}_{X}(x) \mathrm{d} x .
\end{gathered}
$$

Thus, many results about $X_{\mathrm{ew}}$ can be derived from results about $X_{\mathrm{ttt}}$, and vice versa. We call a non-negative random variable with quantile function $T_{n}^{X}(p)$ the observed TTT random variable of order $n$ of $X$, denoted by $X_{\mathrm{ttt}}^{(n)}$. Evidently, the random variable $X_{n}$ in Nair et al. [26] is really the $X_{\mathrm{ttt}}^{(n)}$, the observed TTT random variable of order $n$ of $X$. Hence, from Definition 1, we see that

$$
X \leq_{\mathrm{ttt}-n} Y \Leftrightarrow X_{n} \leq_{\mathrm{ttt}} Y_{n} \Leftrightarrow X_{\mathrm{ttt}}^{(n)} \leq_{\mathrm{ttt}} X_{\mathrm{ttt}}^{(n)} \Leftrightarrow X_{\mathrm{ttt}}^{(n+1)} \leq_{\mathrm{st}} X_{\mathrm{ttt}}^{(n+1)},
$$

with

$$
X \leq_{\mathrm{ttt}} Y \Leftrightarrow X \leq_{\mathrm{ttt}-0} Y \Leftrightarrow X_{0} \leq_{\mathrm{ttt}} Y_{0} \Leftrightarrow X_{\mathrm{ttt}}^{(0)} \leq_{\mathrm{ttt}} X_{\mathrm{ttt}}^{(0)} \Leftrightarrow X_{\mathrm{ttt}} \leq_{\mathrm{st}} X_{\mathrm{ttt}},
$$

as a special case of $X \leq_{\mathrm{ttt}-n} Y$ order, where $X_{0}=X=X_{\mathrm{ttt}}^{(0)}$, the observed TTT random variable of order 0 of $X$.

Theorem 2. If $\theta \geq 1$, then $X \leq_{t t t-n} \theta X$, and if $0<\theta \leq 1$, then $\theta X \leq_{t t t-n} X$.

Theorem 3. Let $\theta$ be a positive real number. Assume that $X \leq_{t t t-n} Y$.

(a) If $\theta \geq 1$, then $X \leq_{t t t-n} \theta Y$.

(b) If $0<\theta \leq 1$, then $\theta X \leq_{t t t-n} Y$. 
Proof. We give proof for (a); the proof for (b) is similar. Suppose that $X \leq_{t t t-n} Y$, then, from Corollary 1, we have

$$
\int_{0}^{p}(1-u)^{n+1}\left[q_{Y}(u)-q_{X}(u)\right] \mathrm{d} u \geq 0, \quad \text { for all } p \in[0,1] .
$$

Furthermore, from Corollary $1, X \leq_{\mathrm{ttt}-n} \theta Y$ if and only if

$$
\int_{0}^{p}(1-u)^{n+1}\left[q_{\theta Y}(u)-q_{X}(u)\right] \mathrm{d} u \geq 0, \quad \text { for all } p \in[0,1] .
$$

It is not hard to see that $q_{\theta Y}(u)=\theta q_{Y}(u)$. On using (13) if $\theta \geq 1$, we get, for all $p \in[0,1]$,

$$
\int_{0}^{p}(1-u)^{n+1}\left[q_{\theta Y}(u)-q_{X}(u)\right] \mathrm{d} u \geq \int_{0}^{p}(1-u)^{n+1}\left[q_{Y}(u)-q_{X}(u)\right] \mathrm{d} u .
$$

Making use of (12), we know that (13) holds. Thus, the stated result follows.

From Theorem 3, we have the following corollary.

Corollary 2. Let $a$ and $b$ be any real numbers such that $0<a \leq b$. If $X \leq_{t t t-n} Y$, then $a X \leq_{t t t-n} b Y$.

Now, we recall the dispersive order (see Shaked and Shanthikumar [31]). Assume that $X$ and $Y$ are two non-negative and continuous random variables with, respectively, distribution functions $F_{X}$ and $G_{Y}$, density functions $f_{X}$ and $g_{Y}$, quantile functions $F_{X}^{-1}$ and $G_{Y}^{-1} . X$ is said to be smaller than $Y$ in the dispersive order (denoted by $X \leq_{\operatorname{disp}} Y$ ) if

$$
F_{X}^{-1}\left(p_{2}\right)-F_{X}^{-1}\left(p_{1}\right) \leq G_{Y}^{-1}\left(p_{2}\right)-G_{Y}^{-1}\left(p_{2}\right), \quad \text { for } 0<p_{1} \leq p_{2}<1 .
$$

That is,

$$
\int_{p_{1}}^{p_{2}} q_{X}(u) \mathrm{d} u \leq \int_{p_{1}}^{p_{2}} q_{Y}(u) \mathrm{d} u, \quad \text { for } 0<p_{1} \leq p_{2}<1 .
$$

Equivalently,

$$
f_{X}\left[F_{X}^{-1}(p)\right] \geq g_{Y}\left[G_{Y}^{-1}(p)\right], \quad \text { for all } p \in(0,1),
$$

equivalently,

$$
q_{X}(p) \leq g_{Y}(p), \quad \text { for all } p \in(0,1)
$$

or equivalently,

$$
\frac{f_{X}(x)}{g_{Y}\left[G_{Y}^{-1}\left(F_{X}(x)\right)\right]} \geq 1, \quad \text { for all } x \geq 0 .
$$

From (16) and Corollary 1, we have the following theorem, which gives a sufficient condition for the $\leq_{\mathrm{ttt}-n}$ order.

Theorem 4. If $X \leq_{\text {disp }} Y$, then $X \leq_{t t t-n} Y$.

Example 1. Let $X$ and $Y$ be two exponential random variables with respective parameters $\lambda_{1}>0$ and $\lambda_{2}>0$. Then, the survival functions functions of $X$ and $Y$ are given by

$$
\bar{F}_{X}(x)=e^{-\lambda_{1} x} \text { and } \bar{G}_{Y}(x)=e^{-\lambda_{2} x}, \text { for all } x \geq 0 .
$$

One can check that

$$
\frac{f_{X}(x)}{g_{Y}\left[G_{Y}^{-1}\left(F_{X}(x)\right)\right]}=\frac{\lambda_{1}}{\lambda_{2}}, \text { for all } x \geq 0 \text {. }
$$


Then, the TTT-n ordering is determined by the parameters $\lambda_{1}$ and $\lambda_{2}$ :

(1) If $\lambda_{1} \geq \lambda_{2}>0$, making use of (18) and (17) we have $X \leq_{\text {disp }} Y$. By Theorem 2, we find that $X \leq_{t t t-n} Y$.

(2) If $0<\lambda_{1} \leq \lambda_{2}$, on using a similar manner as above, we have $Y \leq_{\text {disp }} X$. Again, by Theorem 2, we find that $Y \leq_{t t t-n} X$.

Example 2. Let $X$ and $Y$ be two Pareto random variables with respective survival functions

$$
\bar{F}_{X}(x)=\left(\frac{\lambda}{\lambda+x}\right)^{\alpha_{1}} \text { and } \bar{G}_{Y}(x)=\left(\frac{\lambda}{\lambda+x}\right)^{\alpha_{2}}, \text { for all } x \geq 0 \text {. }
$$

where $\alpha_{1}, \alpha_{2}, \lambda$ are positive real numbers. It can be verified that

$$
\frac{f_{X}(x)}{g_{Y}\left[G_{Y}^{-1}\left(F_{X}(x)\right)\right]}=\frac{\alpha_{1}}{\alpha_{2}}\left(1+\frac{x}{\lambda}\right)^{\frac{\alpha_{1}}{\alpha_{2}}-1}, \text { for all } x \geq 0
$$

Then, the TTT- $n$ ordering is determined by the shape parameters $\alpha_{1}$ and $\alpha_{2}$ :

(1) If $\alpha_{1} \geq \alpha_{2}>0$, in view of (19) and (17), one can see that $X \leq_{\text {disp }} Y$. By Theorem 2, we find that $X \leq_{t t t-n} Y$.

(2) If $0<\alpha_{1} \leq \alpha_{2}$, with a similar pattern as above we get $Y \leq{ }_{\text {disp }} X$. By Theorem 2, we find that $Y \leq_{t t t-n} X$.

Example 3. Let $X$ and $Y$ be two uniform random variables with distribution functions, respectively,

$$
F_{X}(x)=x \quad \text { for all } x \in(0,1) \quad \text { and } \quad G_{Y}(x)=x / 2 \text { for all } x \in(0,2) \text {. }
$$

We can prove that

$$
\frac{f_{X}(x)}{g_{Y}\left[G_{Y}^{-1}\left(F_{X}(x)\right)\right]}=2, \quad \text { for all } x \in(0,1)
$$

In view of (17) and (20), we see that $X \leq_{\text {disp }} Y$. From Theorem 2 we find that $X \leq_{t t t-n} Y$.

Recall from Shaked and Shanthikumar [31] that $X$ is said to be smaller than $Y$ in the increasing concave order (denoted by $X \leq_{\mathrm{icv}} Y$ ) if

$$
\int_{0}^{t} \bar{F}_{X}(x) \mathrm{d} x \leq \int_{0}^{t} \bar{G}_{Y}(x) \mathrm{d} x, \quad \text { for all } t \geq 0
$$

Equivalently,

$$
\int_{0}^{t}\left[F_{X}(x)-G_{Y}(x)\right] \mathrm{d} x \geq 0, \quad \text { for all } t \geq 0 .
$$

Kochar et al. [20] (also see Shaked and Shanthikumar [31]) showed that if $X$ and $Y$ have zero as the common left endpoint of their supports, then

$$
X \leq_{\mathrm{ttt}} Y \Longrightarrow X \leq_{\mathrm{icv}} Y
$$

Nair et al. [26] showed that

$$
X \leq_{\mathrm{ttt}} Y \Longrightarrow X \leq_{\mathrm{ttt}-n} Y
$$

The following proposition considers the implication relationships between the orders $\leq_{\mathrm{ttt}-n}$ and $\leq_{\mathrm{icv}}$. 
Proposition 1. Let $X$ and $Y$ be two non-negative continuous random variables having 0 as the common left endpoint of their interval supports. Then, the orders $\leq_{t t t-n}$ and $\leq_{\mathrm{icv}}$ do not coincide with each other. That is,

$$
X \leq_{t t t-n} Y \nRightarrow X \leq_{\mathrm{icv}} Y \text { and } X \leq_{\mathrm{icv}} Y \nRightarrow X \leq_{t t t-n} Y .
$$

The following two counterexamples show the correctness of Proposition 1. Counterexample 1 reveals $\leq_{\mathrm{ttt}-n} \nRightarrow \leq_{\mathrm{icv}}$; Furthermore, Counterexample 2 indicates $\leq_{\mathrm{icv}} \nRightarrow \leq_{\mathrm{ttt}-n}$.

Counterexample 1. Let $X$ and $Y$ be two non-negative continuous random variables with distribution functions, respectively,

$$
F_{X}(x)=x, x \in(0,1) \quad \text { and } \quad G_{Y}(x)= \begin{cases}\frac{2}{3} x, & x \in\left[0, \frac{3}{8}\right] \\ 3 x-\frac{7}{8}, & x \in\left(\frac{3}{8}, \frac{1}{2}\right] \\ \frac{3}{4} x+\frac{1}{4}, & x \in\left(\frac{1}{2}, 1\right]\end{cases}
$$

One can verify by Theorem 1 that $X \leq_{\mathrm{ttt}-2} Y$. In fact, a straightforward calculation gives

$$
\frac{f_{X}(x)}{g_{Y}\left[G_{Y}^{-1}\left(F_{X}(x)\right)\right]}= \begin{cases}\frac{3}{2}, & x \in\left[0, \frac{1}{4}\right] \\ \frac{1}{3}, & x \in\left(\frac{1}{4}, \frac{5}{8}\right] \\ \frac{4}{3}, & x \in\left(\frac{5}{8}, 1\right]\end{cases}
$$

Denote the function

$$
I_{2}(t)=\int_{0}^{t}\left[\bar{F}_{X}(x)\right]^{3}\left[\frac{f_{X}(x)}{g_{Y}\left[G_{Y}^{-1}\left(F_{X}(x)\right)\right]}-1\right] \mathrm{d} x, \quad \text { for all } t \geq 0 .
$$

From (24), we find that:

(1) when $t \in\left[0, \frac{1}{4}\right]$,

$$
I_{2}(t)=\frac{1}{2} \int_{0}^{t}(1-x)^{3} \mathrm{~d} x \geq 0 .
$$

(2) when $t \in\left(\frac{1}{4}, \frac{5}{8}\right]$,

$$
I_{2}(t)=\frac{1}{2} \int_{0}^{\frac{1}{4}}(1-x)^{3} \mathrm{~d} x-\frac{2}{3} \int_{\frac{1}{4}}^{t}(1-x)^{3} \mathrm{~d} x=\frac{67}{2048}+\frac{1}{6}(1-t)^{4} \geq 0 .
$$

(3) when $t \in\left(\frac{5}{8}, 1\right]$,

$$
I_{2}(t)=I_{2}\left(\frac{5}{8}\right)+\frac{1}{3} \int_{\frac{5}{8}}^{1}(1-x)^{3} \mathrm{~d} x \geq I_{2}\left(\frac{5}{8}\right)=\frac{295}{8192}>0 .
$$

(4) when $t>1, I_{2}(t) \geq 0$ trivially holds.

Hence, by using Theorem 1 , we obtain $X \leq_{\mathrm{ttt}-2} Y$.

Moreover, denote the function

$$
I_{1}(t)=\int_{0}^{t}\left[F_{X}(x)-G_{Y}(x)\right] \mathrm{d} x, \quad \text { for all } t \geq 0 .
$$

In view of (25), when $t=\frac{3}{8}$,

$$
I_{1}\left(\frac{3}{8}\right)=\int_{0}^{\frac{3}{8}}\left[F_{X}(x)-G_{Y}(x)\right] \mathrm{d} x=\int_{0}^{\frac{3}{8}}\left(x-\frac{2}{3} x\right) \mathrm{d} x=\frac{3}{128}>0 ;
$$


when $t=1$,

$$
I_{1}(1)=\int_{0}^{1}\left[F_{X}(x)-G_{Y}(x)\right] \mathrm{d} x=I_{1}\left(\frac{3}{8}\right)+\int_{\frac{3}{8}}^{\frac{1}{2}}\left(\frac{7}{8}-2 x\right) \mathrm{d} x+\frac{1}{4} \int_{\frac{1}{2}}^{1}(x-1) \mathrm{d} x=-\frac{1}{128}<0 .
$$

Making using of Theorem 1, this fact shows that $X \not \not_{\mathrm{icx}} Y$ and $Y \not \mathbf{X}_{\mathrm{icx}} X$.

Counterexample 2. Let $X$ and $Y$ be two non-negative continuous random variables with distribution functions, respectively,

$$
F_{X}(x)=x, x \in[0,1] \quad \text { and } \quad G_{Y}(x)= \begin{cases}\frac{1}{5} x, & x \in\left[0, \frac{5}{8}\right] \\ 6 x-\frac{29}{8}, & x \in\left(\frac{5}{8}, \frac{3}{4}\right] \\ \frac{1}{2} x+\frac{1}{2}, & x \in\left(\frac{3}{4}, 1\right]\end{cases}
$$

Denote the function

$$
I_{1}(t)=\int_{0}^{t}\left[F_{X}(x)-G_{Y}(x)\right] \mathrm{d} x, \quad \text { for all } t \geq 0 .
$$

In view of (26), when $t \in\left[0, \frac{5}{8}\right]$,

$$
I_{1}(t)=\int_{0}^{t}\left[F_{X}(x)-G_{Y}(x)\right] \mathrm{d} x=\int_{0}^{t}\left(x-\frac{1}{5} x\right) \mathrm{d} x=\frac{2}{5} t^{2} \geq 0 ;
$$

when $t \in\left(\frac{5}{8}, \frac{3}{4}\right]$,

$$
I_{1}(t)=I_{1}\left(\frac{5}{8}\right)+\int_{\frac{3}{4}}^{t}\left[F_{X}(x)-G_{Y}(x)\right] \mathrm{d} x=\frac{5}{32}+\int_{\frac{5}{8}}^{t}\left(\frac{29}{8}-5 x\right) \mathrm{d} x=\frac{29}{8} t-\frac{5}{2} t^{2}-\frac{165}{128} \geq 0 ;
$$

when $t \in\left(\frac{3}{4}, 1\right]$,

$$
I_{1}(t)=I_{1}\left(\frac{3}{4}\right)+\int_{\frac{3}{4}}^{t}\left[F_{X}(x)-G_{Y}(x)\right] \mathrm{d} x=\frac{3}{128}+\frac{1}{2} \int_{\frac{3}{4}}^{t}(x-1) d x=\frac{33}{128}+\frac{1}{4} t^{2}-\frac{1}{2} t \geq 0 .
$$

By means of (21) we obtain $X \leq_{\mathrm{icv}} Y$.

Furthermore, denote the function

$$
I_{2}(t)=\int_{0}^{t}\left[\bar{F}_{X}(x)\right]^{3}\left[\frac{f_{X}(x)}{g_{Y}\left[G_{Y}^{-1}\left(F_{X}(x)\right)\right]}-1\right] \mathrm{d} x, \quad \text { for all } t \geq 0 .
$$

From (27) we have, when $t=\frac{1}{8}$,

$$
I_{2}\left(\frac{1}{8}\right)=4 \int_{0}^{\frac{1}{8}}(1-x)^{3} \mathrm{~d} x=\frac{1695}{4096}>0 .
$$

When $t=\frac{7}{8}$,

$I_{2}\left(\frac{7}{8}\right)=I_{2}\left(\frac{1}{8}\right)-\frac{5}{6} \int_{\frac{1}{8}}^{\frac{7}{8}}(1-x)^{3} \mathrm{~d} x=\frac{1695}{16,384}-\frac{5}{6} \int_{\frac{1}{8}}^{\frac{7}{8}}(1-x)^{3} \mathrm{~d} x=-\frac{305}{16,384}<0$.

By using Theorem 1, this fact shows that $X \not_{\mathrm{ttt}-2} Y$ and $Y \not_{\mathrm{ttt}-2} X$. Nair et al. [26] showed that

$$
X \leq_{\mathrm{ttt}} Y \Rightarrow X \leq_{\mathrm{ttt}-n} Y .
$$


The following remark considers whether the inverse of this proposition holds. The answer is negative.

Remark 1. Let $X$ and $Y$ be two non-negative continuous random variables having a common left endpoint of their interval supports. Then

$$
X \leq_{t t t-n} Y \nRightarrow X \leq_{t t t} Y .
$$

This is so, because if $X \leq_{\mathrm{ttt}-n} Y \Rightarrow X \leq_{\mathrm{ttt}} Y$, from (22) we have $X \leq_{\mathrm{ttt}-n} Y \Rightarrow X \leq_{\mathrm{icv}} Y$. However, this result contradicts Proposition 1.

A random variable $X$ is said to be smaller than another random variable $Y$ in the convex transform order (denoted by $X \leq_{\mathrm{c}} Y$ ) if the function $G_{Y}^{-1}\left[F_{X}(x)\right]$ is convex, equivalently, $f_{X}(x) / g_{Y}\left[G_{Y}^{-1}\left(F_{X}(x)\right)\right]$ is increasing in $x \geq 0$.

Theorem 5. Let $X$ and $Y$ be two absolutely continuous non-negative random variables with 0 as the common left endpoint of their supports. Assume that $f_{X}(x) \geq g_{Y}(0)>0$. If $X \leq_{c} Y$, then $X \leq_{t t t-n} Y$.

Proof. In view of (9), $X \leq_{\mathrm{ttt}-n} Y$ if and only if

$$
\int_{0}^{t}\left[\bar{F}_{X}(x)\right]^{n+1}\left[\frac{f_{X}(x)}{g_{Y}\left[G_{Y}^{-1}\left(F_{X}(x)\right)\right]}-1\right] \mathrm{d} x \geq 0, \quad \text { for all } t \geq 0 .
$$

If $X \leq_{\mathrm{c}} Y$, then, the function $f_{X}(x) / g_{Y}\left[G_{Y}^{-1}\left(F_{X}(x)\right)\right]$ is increasing in $x \geq 0$, and $F_{X}(0)=G_{Y}(0)$, hence

$$
\frac{f_{X}(x)}{g_{Y}\left[G_{Y}^{-1}\left(F_{X}(x)\right)\right]} \geq \frac{f_{X}(0)}{g_{Y}\left[G_{Y}^{-1}\left(F_{X}(0)\right)\right]}=\frac{f_{X}(0)}{g_{Y}(0)}, \quad \text { for all } x \geq 0 .
$$

Since $f_{X}(x) \geq g_{Y}(0)$, on using (31) we obtain that $f_{X}(x) / g_{Y}\left[G_{Y}^{-1}\left(F_{X}(x)\right)\right] \geq 1$. Thus, we know that (30) holds. That is, $X \leq_{\mathrm{ttt}-n} Y$.

Theorem 6. The orders $X \leq_{t t t-n} Y$ and $Y \leq_{t t t-n} X$ hold simultaneously if and only if $X={ }_{d i s p} Y$, here, $X={ }_{\text {disp }} Y$ means that $X{ }_{s t} Y+k$, where $k$ is constant.

Proof. From (9) we have that $X \leq_{\mathrm{ttt}-n} Y$ and $Y \leq_{\mathrm{ttt}-n} X$ hold simultaneously, if and only if

$$
\int_{0}^{t}\left[\bar{F}_{X}(x)\right]^{n+1}\left[\frac{f_{X}(x)}{g_{Y}\left[G_{Y}^{-1}\left(F_{X}(x)\right)\right]}-1\right] \mathrm{d} x \equiv 0, \quad \text { for all } t \geq 0 .
$$

That is,

$$
\frac{f_{X}(x)}{g_{Y}\left[G_{Y}^{-1}\left(F_{X}(x)\right)\right]}=1, \text { almost surely, }
$$

which is equivalent to that $X=\operatorname{disp} Y$. This completes the proof.

\section{Closure and Reversed Closure Properties of the TTT- $n$ Ordering}

3.1. Closure and Reversed Closure Properties of the TTT-n Ordering with Respect to Series and Parallel Systems

Let $X$ and $Y$ be two non-negative and continuous random variables with distribution functions $F_{X}$ and $G_{Y}$, right-continuous inverse functions $F_{X}^{-1}$ and $G_{Y}^{-1}$, survival functions 
$\bar{F}_{X}$ and $\bar{G}_{Y}$, and density functions $f_{X}$ and $g_{Y}$, respectively, $X_{1}, \ldots, X_{m}$ and $Y_{1}, \ldots, Y_{m}$ are independent and identically distributed (i.i.d.) copies of $X$ and $Y$, respectively, denote by

$$
X_{1: m}=\min \left\{X_{1}, \ldots, X_{m}\right\}, \quad X_{m: m}=\max \left\{X_{1}, \ldots, X_{m}\right\} .
$$

Similarly, $Y_{1: m}$ and $Y_{m: m}$ denote the survival function and the density function of $X_{1: m}$ by $\bar{F}_{X_{1: m}}$ and $f_{X_{1: m}}$, respectively; denote the distribution function and the density function of $X_{m: m}$ by $F_{X_{m: m}}$ and $f_{X_{m: m} \prime}$, respectively. Similarly, $\bar{G}_{Y_{1: m}}, g_{Y_{1: m}}, G_{Y_{n: m}}$, and $g_{Y_{m: m}}$.

A series system is such a system that functions if and only if each component functions. A parallel system is such a system that functions if and only if at least one component functions. Then, a series system composed of $m$ i.i.d. components has its lifetime $X_{1: m}$; a parallel system composed of $m$ i.i.d. components has its lifetime $X_{m: m}$.

Below, we consider the reversed closure property of a parallel system for TTT- $n$ ordering, we have the following result.

Theorem 7. If $X_{m: m} \leq_{t t t-n} Y_{m: m}$, then $X \leq_{t t t-n} Y$.

Proof. Suppose that $X_{m: m} \leq_{t t t-n} Y_{m: m}$. Then, from (9) we obtain

$$
\int_{0}^{t}\left[\bar{F}_{X_{m: m}}(x)\right]^{n+1}\left[\frac{f_{X_{m: m}}(x)}{g Y_{m: m}\left[G_{Y_{m: m}}^{-1}\left(F_{X_{m: m}}(x)\right)\right]}-1\right] \mathrm{d} x \geq 0, \quad \text { for all } t \geq 0 .
$$

Since $F_{X_{m: m}}(x)=\left[F_{X}(x)\right]^{m}$, by noting that Shaked and Shanthikumar [18] showed that

$$
G_{Y_{m: m}}^{-1}\left[F_{X_{m: m}}(x)\right]=G_{Y}^{-1}\left[F_{X}(x)\right], \quad \text { for all } x \geq 0,
$$

it can be proven that

$$
\frac{f_{X_{m: m}}(x)}{g_{Y_{m: m}}\left[G_{Y_{m: m}}^{-1}\left(F_{X_{m: m}}(x)\right)\right]}=\frac{f_{X}(x)}{g_{Y}\left[G_{Y}^{-1}\left(F_{X}(x)\right)\right]}, \text { for all } x \geq 0 .
$$

It is easy to see that $\bar{F}_{X_{m: m}}(x)=\bar{F}_{X}(x)\left[\sum_{i=1}^{m}\left[F_{X}(x)\right]^{i-1}\right]$. On using (32) and (33), we find that

$$
\int_{0}^{t}\left[\bar{F}_{X}(x)\right]^{n+1}\left[\sum_{i=1}^{m}\left[F_{X}(x)\right]^{i-1}\right]^{n+1}\left[\frac{f_{X}(x)}{g_{Y}\left[G_{Y}^{-1}\left(F_{X}(x)\right)\right]}-1\right] \mathrm{d} x \geq 0, \quad \text { for all } t \geq 0 .
$$

Obviously, the function $\left[\sum_{i=1}^{m}\left[F_{X}(x)\right]^{i-1}\right]^{-(n+1)}$ is non-negative and decreasing in $x \geq 0$, making use of (34) and Lemma 1 , we have

$$
\int_{0}^{t}\left[\bar{F}_{X}(x)\right]^{n+1}\left[\frac{f_{X}(x)}{g_{Y}\left[G_{Y}^{-1}\left(F_{X}(x)\right)\right]}-1\right] \mathrm{d} x \geq 0, \quad \text { for all } t \geq 0 .
$$

Again, by (9), this completes the proof.

Remark 2. Theorem 7 indicates that the TTT-n ordering has the reversed closure property under the formation of parallel systems. Theorem 7 also says that the parallel systems reversely preserve the TTT- $n$ ordering.

Remark 3. The inverse of Theorem 7 is not necessarily true. That is,

$$
X \leq_{t t t-n} Y \nRightarrow X_{m: m} \leq_{t t t-n} Y_{m: m}
$$


To see this relationship, we give the following counterexample.

Counterexample 3. Let $X$ and $Y$ be two non-negative continuous random variables with distribution functions, respectively,

$$
F_{X}(x)=x, x \in(0,1) \quad \text { and } \quad G_{Y}(x)= \begin{cases}\frac{2}{3} x, & x \in\left[0, \frac{3}{8}\right] ; \\ 3 x-\frac{7}{8}, & x \in\left(\frac{3}{8}, \frac{1}{2}\right] \\ \frac{3}{4} x+\frac{1}{4}, & x \in\left(\frac{1}{2}, 1\right] .\end{cases}
$$

A simple computation yields

$$
\frac{f_{X}(x)}{g_{Y}\left[G_{Y}^{-1}\left(F_{X}(x)\right)\right]}= \begin{cases}\frac{3}{2}, & x \in\left[0, \frac{1}{4}\right] \\ \frac{1}{3}, & x \in\left(\frac{1}{4}, \frac{5}{8}\right] \\ \frac{4}{3}, & x \in\left(\frac{5}{8}, 1\right] .\end{cases}
$$

From Counterexample 1, we know that $X \leq_{\mathrm{ttt}-2} Y$.

Furthermore, one can easily testify that

$$
\frac{f_{X_{m: m}}(x)}{g_{Y_{m: m}}\left[G_{Y_{m: m}}^{-1}\left(F_{X_{m: m}}(x)\right)\right]}=\frac{f_{X}(x)}{g_{Y}\left[G_{Y}^{-1}\left(F_{X}(x)\right)\right]}, \quad \text { for all } x \geq 0 .
$$

Note that when $m=2, \bar{F}_{X_{2: 2}}(x)=1-\left[F_{X}(x)\right]^{2}=1-x^{2}, x \in[0,1]$. From (9), when $n=2$, denote the function

$$
\begin{aligned}
I_{p}(t) & =\int_{0}^{t}\left[\bar{F}_{X_{2: 2}}(x)\right]^{2+1}\left[\frac{f_{X_{2: 2}}(x)}{g_{Y_{2: 2}}\left[G_{Y_{2: 2}}^{-1}\left(F_{X_{2: 2}}(x)\right)\right]}-1\right] \mathrm{d} x, \quad \text { for all } t \geq 0 \\
& = \begin{cases}\int_{0}^{t}\left(1-x^{2}\right)^{3}\left[\frac{f_{X}(x)}{g_{Y}\left[G_{Y}^{-1}\left(F_{X}(x)\right)\right.}-1\right] \mathrm{d} x, & x \in[0,1] ; \\
I_{p}(1), & x \in(1,+\infty) .\end{cases}
\end{aligned}
$$

Hence, from (36), we find that

(1) when $t \in\left[0, \frac{1}{4}\right]$, since $1-x^{2} \geq 0, x \in\left[0, \frac{1}{4}\right]$, therefore

$$
I_{p}(t)=\frac{1}{2} \int_{0}^{t}\left[1-x^{2}\right]^{3} \mathrm{~d} x \geq 0 .
$$

(2) when $t \in\left(\frac{1}{4}, \frac{5}{8}\right]$,

$$
I_{p}(t)=I_{p}\left(\frac{1}{4}\right)-\frac{2}{3} \int_{\frac{1}{4}}^{t}\left[1-x^{2}\right]^{3} \mathrm{~d} x .
$$

A direct calculation shows that $I_{p}\left(\frac{5}{8}\right)=-1.4394 \times 10^{-2}<0$. Making use of Theorem 1 , this fact shows that $X_{2: 2} \mathbb{E}_{\mathrm{ttt}-n} Y_{2: 2}$ and $Y_{2: 2} \mathbb{E}_{\mathrm{ttt}-n} X_{2: 2}$. Thus, we conclude that $X \leq_{\mathrm{ttt}-n}$ $Y \nRightarrow X_{m: m} \leq_{\mathrm{ttt}-n} Y_{m: m}$.

Nair et al. ([26], p. 1137, Theorem 5.1) showed the following result, for the ease of citation we list as a lemma, and we give a new proof. Here, we point out that the component numbers of systems are not the same as the order $n$ of the TTT- $n$ ordering generally.

Lemma 2 (Nair et al. [26]). If $X \leq_{t t t-n} Y$, then $X_{1: n} \leq_{t t t-n} Y_{1: n}$.

Theorem 8. If $X \leq_{t t t-n} Y$, then $X_{1: m} \leq_{t t t-n} Y_{1: m}$. 
Proof. Suppose that $X \leq_{\mathrm{ttt}-n} Y$. Then, from (9) we have,

$$
\int_{0}^{t}\left[\bar{F}_{X}(x)\right]^{n+1}\left[\frac{f_{X}(x)}{g_{Y}\left[G_{Y}^{-1}\left(F_{X}(x)\right)\right]}-1\right] \mathrm{d} x \geq 0, \quad \text { for all } t \geq 0 .
$$

Note that $\bar{F}_{X_{1: m}}(x)=\left[\bar{F}_{X}(x)\right]^{m}$ and $\bar{G}_{Y_{1: m}}(x)=\left[\bar{G}_{Y}(x)\right]^{m}$. It is easy to prove that

$$
G_{Y_{1: m}}^{-1}\left(F_{X_{1: m}}(x)\right)=G_{Y}^{-1}\left(F_{X}(x)\right) \text {. }
$$

Differentiating both sides of this equation, we find that

$$
\frac{f_{X_{1: m}}(x)}{g_{Y_{1: m}}\left[G_{Y_{1: m}}^{-1}\left(F_{X_{1: m}}(x)\right)\right]}=\frac{f_{X}(x)}{g_{Y}\left[G_{Y}^{-1}\left(F_{X}(x)\right)\right]}, \quad \text { for all } x \geq 0 .
$$

Since the function

$$
h(x)=\left[\frac{\bar{F}_{X_{1: m}}(x)}{\bar{F}_{X}(x)}\right]^{n+1}=\left[\left(\bar{F}_{X}(x)\right)^{m-1}\right]^{n+1}
$$

is non-negative and decreasing in $x \geq 0$, by (37)-(39) and Lemma 1 , we find that

$$
\int_{0}^{t}\left[\bar{F}_{X_{1: m}}(x)\right]^{n+1}\left[\frac{f_{X_{1: m}}(x)}{g Y_{1: m}\left[G_{Y_{1: m}}^{-1}\left(F_{X_{1: m}}(x)\right)\right]}-1\right] \mathrm{d} x \geq 0,
$$

again, by using Theorem 1 , which is equivalent to $X_{1: m} \leq_{\mathrm{ttt}-n} Y_{1: m}$. This completes the proof.

Remark 4. Theorem 8 indicates that the series systems preserve the TTT-n ordering. Theorem 7 also says that the TTT-n ordering has the closure property with respect to the series systems.

Remark 5. The inverse of Theorem 8 is not necessarily true. To see this relationship, we give the following counterexample.

Counterexample 4. Let $X$ and $Y$ be two non-negative continuous random variables with distribution functions, respectively,

$$
F_{X}(x)=x, x \in(0,1) \quad \text { and } \quad G_{Y}(x)= \begin{cases}\frac{1}{2} x, & x \in\left[0, \frac{1}{8}\right] \\ \frac{3}{2} x-\frac{1}{8}, & x \in\left(\frac{1}{8}, \frac{3}{4}\right] .\end{cases}
$$

On using (38), we have

$$
\frac{f_{X_{1: 2}}(x)}{g_{Y_{1: 2}}\left[G_{Y_{1: 2}}^{-1}\left(F_{X_{1: 2}}(x)\right)\right]}=\frac{f_{X}(x)}{g_{Y}\left[G_{Y}^{-1}\left(F_{X}(x)\right)\right]}, \quad \text { for all } x \geq 0 .
$$

One can testify by Theorem 1 that $X_{1: 2} \leq_{\mathrm{ttt}-2} Y_{1: 2}$. In fact, a direct computation yields

$$
\frac{f_{X}(x)}{g_{Y}\left[G_{Y}^{-1}\left(F_{X}(x)\right)\right]}=\left\{\begin{array}{cc}
2, & x \in\left[0, \frac{1}{16}\right] \\
\frac{2}{3}, & x \in\left(\frac{1}{16}, 1\right] .
\end{array}\right.
$$


Note that $\bar{F}_{X_{1: 2}}(x)=\left[\bar{F}_{X}(x)\right]^{2}$. Due to (9), when $n=2$, let

$$
\begin{aligned}
& I_{S}(t)=\int_{0}^{t}\left[\bar{F}_{X_{1: 2}}(x)\right]^{2+1}\left[\frac{f_{X_{1: 2}}(x)}{g_{Y_{1: 2}}\left[G_{Y_{1: 2}}^{-1}\left(F_{X_{1: 2}}(x)\right)\right]}-1\right] \mathrm{d} x \\
& =\int_{0}^{t}\left[\bar{F}_{X}(x)\right]^{6}\left[\frac{f_{X}(x)}{g_{Y}\left[G_{Y}^{-1}\left(F_{X}(x)\right)\right]}-1\right] \mathrm{d} x, \quad \text { for all } t \geq 0 .
\end{aligned}
$$

From (43), we get

(1) When $t \in\left[0, \frac{1}{16}\right]$,

$$
I_{S}(t)=\frac{1}{2} \int_{0}^{t}(1-x)^{6} \mathrm{~d} x=\frac{1}{7}\left[1-(1-t)^{7}\right] \geq 0 .
$$

(2) When $t \in\left(\frac{1}{16}, 1\right]$,

$$
\begin{gathered}
I_{S}(t)=I_{S}\left(\frac{1}{16}\right)-\frac{1}{3} \int_{0}^{t}(1-x)^{6} \mathrm{~d} x=\frac{1}{7}-\frac{4}{21}\left(\frac{15}{16}\right)^{7}+\frac{1}{12}(1-t)^{7} \\
\geq \frac{1}{7}-\frac{4}{21}\left(\frac{15}{16}\right)^{7} \approx 2.1619 \times 10^{-2}>0 .
\end{gathered}
$$

When $t>1, I_{s}(t) \geq 0$ trivially holds. Hence, by using Theorem 1 , we find that $X_{1: 2} \leq_{\text {ttt-2 }} Y_{1: 2}$.

Moreover, also from (9), when $n=2$, let

$$
I(t)=\int_{0}^{t}\left[\bar{F}_{X}(x)\right]^{3}\left[\frac{f_{X}(x)}{g_{Y}\left[G_{Y}^{-1}\left(F_{X}(x)\right)\right]}-1\right] \mathrm{d} x, \quad \text { for all } t \geq 0 .
$$

In view of (42), when $t=\frac{1}{16}$,

$$
I\left(\frac{1}{16}\right)=\int_{0}^{\frac{1}{16}}\left[\bar{F}_{X}(x)\right]^{3} \mathrm{~d} x=\int_{0}^{\frac{1}{16}}(1-x)^{3} \mathrm{~d} x=\frac{1}{4}\left[1-\left(\frac{15}{16}\right)^{4}\right]>0 ;
$$

when $t=1$,

$$
I(1)=I\left(\frac{1}{16}\right)-\frac{1}{3} \int_{\frac{1}{16}}^{1}(1-x)^{3} \mathrm{~d} x=\frac{1}{4}-\frac{1}{3}\left(\frac{15}{16}\right)^{4} \approx-7.4921 \times 10^{-3}<0 .
$$

Making use of Theorem 1, this fact shows that $X \not_{\mathrm{ttt}-2} Y$ and $Y \not \mathbb{t t t}_{\mathrm{tt}} X$. Therefore, we conclude that $X_{1: m} \leq_{\mathrm{ttt}-n} Y_{1: m} \nRightarrow X \leq_{\mathrm{ttt}-n} Y$.

\subsection{Closure and Reversed Closure Properties of the TTT-n Ordering with Respect to Random Series and Parallel Systems}

Let $X_{1}, X_{2}, \ldots$ and $Y_{1}, Y_{2}, \ldots$ be sequences of i.i.d. copies of random variables $X$ and $Y$, respectively. Let $M$ be a positive integer-valued random variable with the probability mass function $p_{M}(m)=\mathbb{P}\{M=m\}, m=1,2, \ldots$. Assume that $M$ is independent of $X_{i}^{\prime} \mathrm{s}$ and $Y_{i}^{\prime}$ 's. Denote by

$$
X_{1: M}=\min \left\{X_{1}, \ldots, X_{M}\right\}, \quad Y_{1: M}=\min \left\{Y_{1}, \ldots, Y_{M}\right\} ;
$$

and

$$
X_{M: M}=\max \left\{X_{1}, \ldots, X_{M}\right\}, \quad Y_{M: M}=\max \left\{Y_{1}, \ldots, Y_{M}\right\} .
$$

For the ease of proofs of upcoming results, we give the following lemma, the proof is straightforward and hence omitted here. 
Lemma 3. Let $X$ and $Y$ be two absolutely continuous non-negative random variables with distribution functions $F_{X}$ and $G_{Y}$, right-continuous inverse functions $F_{X}^{-1}$ and $G_{Y}^{-1}$, survival functions $\bar{F}_{X}$ and $\bar{G}_{Y}$, and density functions $f_{X}$ and $g_{Y}$, respectively. Then, for all $x \geq 0$,

$$
\frac{f_{X_{1: M}}(x)}{g Y_{1: M}\left[G_{Y_{1: M}}^{-1}\left(F_{X_{1: M}}(x)\right)\right]}=\frac{f_{X}(x)}{g_{Y}\left[G_{Y}^{-1}\left(F_{X}(x)\right)\right]}
$$

and

$$
\frac{f_{X_{M: M}}(x)}{g_{Y_{M: M}}\left[G_{Y_{M: M}}^{-1}\left(F_{X_{M: M}}(x)\right)\right]}=\frac{f_{X}(x)}{g_{Y}\left[G_{Y}^{-1}\left(F_{X}(x)\right)\right]}
$$

Now we consider to extend the results in Theorems 7 and 8 from a finite number $m$ to a random number $M$ of the component numbers of systems.

The following theorem can be viewed as an extension of Theorem 8 .

Theorem 9. If $X \leq_{t t t-n} Y$, then $X_{1: M} \leq_{t t t-n} Y_{1: M}$.

Proof. Suppose that $X \leq_{\mathrm{ttt}-n} Y$. Then, from (9) we have,

$$
\int_{0}^{t}\left[\bar{F}_{X}(x)\right]^{n+1}\left[\frac{f_{X}(x)}{g_{Y}\left[G_{Y}^{-1}\left(F_{X}(x)\right)\right]}-1\right] \mathrm{d} x \geq 0, \quad \text { for all } t \geq 0 .
$$

The survival function of $X_{1: M}$ is given by

$$
\bar{F}_{X_{1: M}}(x)=\sum_{m=1}^{\infty}\left[\bar{F}_{X}(x)\right]^{m} p_{M}(m)=\bar{F}_{X}(x) \sum_{m=1}^{\infty}\left[\bar{F}_{X}(x)\right]^{m-1} p_{M}(m) .
$$

Thus, we know that the function

$$
h(x)=\frac{\left[\bar{F}_{X_{1: M}}(x)\right]^{n+1}}{\left[\bar{F}_{X}(x)\right]^{n+1}}=\left[\sum_{m=1}^{\infty}\left[\bar{F}_{X}(x)\right]^{m-1} p_{M}(m)\right]^{n+1}
$$

is non-negative and decreasing in $x \geq 0$.

Making use of (45), (47), (48) and Lemma 1, we find that

$$
\int_{0}^{t}\left[\bar{F}_{X_{1: M}}(x)\right]^{n+1}\left[\frac{f_{X_{1: M}}(x)}{g Y_{1: M}\left[G_{Y_{1: M}}^{-1}\left(F_{X_{1: M}}(x)\right)\right]}-1\right] \mathrm{d} x \geq 0, \quad \text { for all } t \geq 0 .
$$

Again, by means of (49) and Theorem 1, we see that $X_{1: M} \leq_{\mathrm{ttt}-n} Y_{1: M}$. This is the desired result.

Remark 6. Theorem 9 indicates that random series systems preserve the TTT- $n$ ordering. Theorem 8 also says that the TTT-n ordering has closure property with respect to random series systems.

The following Theorem 10 can be viewed as an extension of Theorem 7 . The proof is similar to that of Theorem 9 and hence omitted here.

Theorem 10. If $X_{M: M} \leq_{t t t-n} Y_{M: M}$, then $X \leq_{t t t-n} Y$.

The following is an illustrative example of Theorem 10. 
Example 4. Let $X$ and $Y$ be two exponential random variables with respective survival functions

$$
\bar{F}_{X}(x)=e^{-2 x} \text { and } \bar{G}_{Y}(x)=e^{-x}, \text { for all } x \geq 0 \text {. }
$$

Let $M$ be a positive integer-valued random variable with probability function

$$
\mathbb{P}(M=1)=\mathbb{P}(M=1)=\frac{1}{2} .
$$

One can testify that

$$
\frac{f_{X}(x)}{g_{Y}\left[G_{Y}^{-1}\left(F_{X}(x)\right)\right]}=2, \quad \text { for all } x \geq 0
$$

and

$$
\frac{f_{X_{M: M}}(x)}{g_{Y_{M: M}}\left[G_{Y_{M: M}}^{-1}\left(F_{X_{M: M}}(x)\right)\right]}=\frac{f_{X}(x)}{g_{Y}\left[G_{Y}^{-1}\left(F_{X}(x)\right)\right]}, \text { for all } x \geq 0 .
$$

We have that $X_{M: M}$ and $Y_{M: M}$ have distribution functions, respectively,

$$
F_{X_{M: M}}(x)=\frac{1}{2}\left(e^{-2 x}+e^{-4 x}\right), \quad G_{Y_{M: M}}(x)=\frac{1}{2}\left(e^{-x}+e^{-2 x}\right), \quad \text { for all } x \geq 0 .
$$

Thus,

$$
\bar{F}_{X_{M: M}}(x)=1-\frac{1}{2}\left(e^{-2 x}+e^{-4 x}\right), \text { for all } x \geq 0 .
$$

When $n=2$, denote the function

$$
\begin{aligned}
I_{p}(t) & =\int_{0}^{t}\left[\bar{F}_{X_{M: M}}(x)\right]^{2+1}\left[\frac{f_{X_{M: M}}(x)}{g_{Y_{M: M}}\left[G_{Y_{M: M}^{-1}}^{-1}\left(F_{X_{M: M}}(x)\right)\right]}-1\right] d x \\
& =\int_{0}^{t}\left[1-\frac{1}{2}\left(e^{-2 x}+e^{-4 x}\right)\right]^{3} \mathrm{~d} x, \quad \text { for all } t \geq 0 .
\end{aligned}
$$

Since $\left[1-\frac{1}{2}\left(e^{-2 x}+e^{-4 x}\right)\right]^{3} \geq 0$, hence $I_{p}(t) \geq 0$, for all $t \geq 0$. Utilizing Theorem 1 we obtain that $X_{M: M} \leq_{t t t-2} Y_{M: M}$. Therefore, by Theorem 10 we have $X \leq_{t t t-2} Y$. In fact, from Example 1, we see that this result is really correct.

Remark 7. Theorem 10 shows that random parallel systems reversedly preserve the TTT-n ordering. Theorem 10 also states that the TTT-n ordering has reversed closure property with respect to random parallel systems.

Remark 8. The inverse of Theorem 10 is not necessarily true. To see this relationship, we give a counterexample below.

Counterexample 5. Let $X$ and $Y$ be two non-negative continuous random variables with distribution functions, respectively,

$$
F_{X}(x)=x, x \in[0,1] \quad \text { and } \quad G_{Y}(x)= \begin{cases}\frac{1}{2} x, & x \in\left[0, \frac{1}{4}\right] \\ \frac{7}{2} x-\frac{3}{4}, & x \in\left(\frac{1}{4}, \frac{1}{2}\right] .\end{cases}
$$

Denote the function

$$
I(t)=\int_{0}^{t}\left[\bar{F}_{X}(x)\right]^{3}\left[\frac{f_{X}(x)}{g_{Y}\left[G_{Y}^{-1}\left(F_{X}(x)\right)\right]}-1\right] \mathrm{d} x, \quad \text { for all } t \geq 0 .
$$


A direct computation yields

$$
\frac{f_{X}(x)}{g_{Y}\left[G_{Y}^{-1}\left(F_{X}(x)\right)\right]}= \begin{cases}2, & x \in\left[0, \frac{1}{8}\right] \\ \frac{2}{7}, & x \in\left(\frac{1}{8}, 1\right] .\end{cases}
$$

For $t \in\left[0, \frac{1}{8}\right]$, we have

$$
I(t)=\int_{0}^{t}(1-x)^{3} \mathrm{~d} x=\frac{1}{4}\left[1-(1-t)^{4}\right] \geq 0 .
$$

For $t \in\left[\frac{1}{8}, 1\right]$, we have

$$
I(t)=I\left(\frac{1}{8}\right)-\frac{5}{7} \int_{\frac{1}{8}}^{t}(1-x)^{3} \mathrm{~d} x=\frac{1}{4}-\frac{3}{7}\left(\frac{3}{4}\right)^{4}+\frac{5}{28}(1-t)^{4} \geq \frac{1}{4}-\frac{3}{7}\left(\frac{3}{4}\right)^{4}=\frac{205}{1792} \geq 0 .
$$

Hence, $I(t) \geq 0$ for all $t \geq 0$. By using Theorem 1 we find that $X \leq_{\mathrm{ttt}-2} Y$.

Now, let $M$ be a geometric random variable with probability function

$$
\mathbb{P}(M=m)=p q^{m-1}, \quad m=1,2, \ldots
$$

Then, $X_{M: M}$ has its distribution function $F_{X_{M: M}}(x)=\frac{F_{X}(x)}{2-F_{X}(x)}$. Thus, the survival function of $X_{M: M}$ is given by $\bar{F}_{X_{M: M}}(x)=2 \cdot \frac{1-F_{X}(x)}{2-F_{X}(x)}$. Denote the function

$$
I_{p}(t)=\int_{0}^{t}\left[\bar{F}_{X_{M: M}}(x)\right]^{3}\left[\frac{f_{X_{M: M}}(x)}{g_{Y_{M: M}}\left[G_{Y_{M: M}}^{-1}\left(F_{X_{M: M}}(x)\right)\right]}-1\right] \mathrm{d} x, \quad \text { for all } t \geq 0 .
$$

Making use of (46), we have

$$
I_{p}\left(\frac{1}{8}\right)=8 \int_{0}^{t}\left(\frac{1-x}{2-x}\right)^{3} \mathrm{~d} x \approx 0.1133>0 ;
$$

and

$$
I_{p}(1)=I_{p}\left(\frac{1}{8}\right)-8 \times \frac{5}{7} \int_{\frac{1}{8}}^{t}\left(\frac{1-x}{2-x}\right)^{3} \mathrm{~d} x=-6.6109 \times 10^{-2}<0 .
$$
That is,

Again, by using Theorem 1, we know that $X_{M: M} \not_{\mathrm{ttt}-n} Y_{M: M}$ and $Y_{M: M} \mathbb{E}_{\mathrm{ttt}-n} X_{M: M}$.

$$
X \leq_{\mathrm{ttt}-n} Y \nRightarrow X_{M: M} \leq_{\mathrm{ttt}-n} Y_{M: M}
$$

3.3. Closure and Reversed Closure Properties of the TTT-n Ordering under Increasing Convex and Concave Transforms

By means of a method used in Kochar et al. [20] we can prove the following Theorems 11 and 12, the details of proofs are omitted here.

Theorem 11. Let $\phi(\cdot)$ be a non-negative and increasing concave function defined on $\Re_{+}=[0, \infty)$ such that $\phi(0)=0$. If $X \leq_{t t t-n} Y$, then $\phi(X) \leq_{t t t-n} \phi(Y)$.

Remark 9. Theorem 11 indicates that the TTT-n ordering has closure property under the concave generalized scale transform. Theorem 11 also says that the TTT-n ordering has closure property under risk aversion transform.

Remark 10. In Theorem 11, the condition " $\phi(\cdot)$ is concave" is only a sufficient condition, but not necessary; see the following counterexample. 
Counterexample 6. Let $X$ and $Y$ be two non-negative continuous random variables with distribution functions, respectively,

$$
F_{X}(x)=x, x \in[0,1] \quad \text { and } \quad G_{Y}(x)= \begin{cases}\frac{2}{3} x, & x \in\left[0, \frac{3}{4}\right] \\ 4 x-\frac{5}{2}, & x \in\left(\frac{3}{4}, \frac{7}{8}\right]\end{cases}
$$

One can calculate that

$$
\frac{f_{X}(x)}{g_{Y}\left[G_{Y}^{-1}\left(F_{X}(x)\right)\right]}= \begin{cases}\frac{3}{2}, & x \in\left[0, \frac{1}{2}\right] ; \\ \frac{1}{4}, & x \in\left(\frac{1}{2}, 1\right] .\end{cases}
$$

In view of (9), when $n=2$, let the function

$$
\begin{aligned}
I(t) & :=\int_{0}^{t}\left[\bar{F}_{X}(x)\right]^{3}\left[\frac{f_{X}(x)}{g_{Y}\left[G_{Y}^{-1}\left(F_{X}(x)\right)\right]}-1\right] \mathrm{d} x \\
& =\int_{0}^{t}(1-x)^{3}\left[\frac{f_{X}(x)}{g_{Y}\left[G_{Y}^{-1}\left(F_{X}(x)\right)\right]}-1\right] \mathrm{d} x, \quad \text { for all } t \geq 0 .
\end{aligned}
$$

When $t \in\left[0, \frac{1}{2}\right]$,

$$
I(t)=\int_{0}^{t} \frac{1}{2}(1-x)^{3} \mathrm{~d} x=\frac{1}{8}\left[1-(1-t)^{4}\right] \geq 0 .
$$

When $t \in\left(\frac{1}{2}, 1\right]$,

$$
I(t)=I\left(\frac{1}{2}\right)-\frac{3}{4} \int_{\frac{1}{2}}^{t}(1-x)^{3} \mathrm{~d} x=I\left(\frac{1}{2}\right)+\frac{3}{16}\left[(1-t)^{4}-\left(\frac{1}{2}\right)^{4}\right] \geq I(1)=\frac{27}{256},
$$

where the inequality is due to the decreasing property of $I(t)$. When $t>1, I(t) \geq 0$ trivially holds. Hence, we get that $I(t) \geq 0$ for all $t \geq 0$. By using Theorem 1 , we see that $X \leq_{\mathrm{ttt}-2} Y$.

Now, take

$$
\phi(x)= \begin{cases}\frac{2}{3} x, & x \in\left[0, \frac{3}{4}\right] \\ 4 x-\frac{5}{2}, & x \in\left(\frac{3}{4}, \frac{7}{8}\right] \\ x+\frac{1}{8}, & x \in\left(\frac{7}{8}, \infty\right) .\end{cases}
$$

One gets that

$$
\phi^{\prime}(x)= \begin{cases}\frac{2}{3}, & x \in\left[0, \frac{3}{4}\right] \\ 4, & x \in\left(\frac{3}{4}, \frac{7}{8}\right] \\ 1, & x \in\left(\frac{7}{8}, \infty\right)\end{cases}
$$

By Theorem 1, we also have

$$
\phi(X) \leq_{\mathrm{ttt}-2} \phi(Y) \Leftrightarrow \int_{0}^{t}\left[\bar{F}_{\phi(X)}(x)\right]^{3}\left[\frac{f_{\phi(X)}(x)}{g_{\phi(Y)}\left[G_{\phi(Y)}^{-1}\left(F_{\phi(X)}(x)\right)\right]}-1\right] \mathrm{d} x \geq 0, \quad \text { for all } t \geq 0 .
$$

Equivalently,

$$
I_{\phi}(t):=\int_{0}^{t} \phi^{\prime}(x)\left[\bar{F}_{X}(x)\right]^{3}\left[\frac{f_{X}(x)}{g_{Y}\left[G_{Y}^{-1}\left(F_{X}(x)\right)\right]} \frac{\phi^{\prime}[\alpha(x)]}{\phi^{\prime}(x)}-1\right] \mathrm{d} x, \quad \text { for all } t \geq 0,
$$


where

$$
\alpha(x):=G_{Y}^{-1}\left[F_{X}(x)\right]= \begin{cases}\frac{3}{2} x, & x \in\left[0, \frac{1}{2}\right] \\ \frac{1}{4} x+\frac{5}{8}, & x \in\left(\frac{1}{2}, 1\right] .\end{cases}
$$

Note that

$$
\phi^{\prime}[\alpha(x)]= \begin{cases}\frac{2}{3}, & x \in\left[0, \frac{1}{2}\right] \\ 4, & x \in\left(\frac{1}{2}, 1\right] \\ 1, & x \in(1, \infty) .\end{cases}
$$

In view of (50), when $t \in\left[0, \frac{1}{2}\right]$, then

$$
I_{\phi}(t)=\frac{1}{3} \int_{0}^{t}(1-x)^{3} \mathrm{~d} x=\frac{1}{12}\left[1-(1-t)^{4}\right] \geq 0 ; \quad I_{\phi}\left(\frac{1}{2}\right)=\frac{5}{64} .
$$

When $t \in\left(\frac{1}{2}, \frac{3}{4}\right]$, then

$$
I_{\phi}(t)=I_{\phi}\left(\frac{1}{2}\right)+\frac{1}{3} \int_{\frac{1}{2}}^{t}(1-x)^{3} \mathrm{~d} x=\frac{1}{12}\left[1-(1-t)^{4}\right] \geq 0 ; \quad I_{\phi}\left(\frac{3}{4}\right)=\frac{85}{1024} .
$$

When $t \in\left(\frac{3}{4}, \frac{7}{8}\right]$, then

$$
\left.I_{\phi}(t)=I_{\phi}\left(\frac{3}{4}\right)-3 \int_{\frac{3}{4}}^{t}(1-x)^{3} \mathrm{~d} x=\frac{1}{12}-\frac{5}{6}\left(\frac{1}{4}\right)^{4}+\frac{3}{4}(1-t)^{4}\right] \geq 0 ; \quad I_{\phi}\left(\frac{3}{4}\right)=\frac{85}{1024} .
$$

When $t \in\left(\frac{7}{8}, 1\right]$, then

$$
I_{\phi}(t)=I_{\phi}\left(\frac{7}{8}\right)=\frac{1}{12}-\frac{5}{6}\left(\frac{1}{4}\right)^{4}+\frac{3}{4}\left(\frac{1}{8}\right)^{4} \geq 0 .
$$

When $t>1, I_{\phi}(t) \geq 0$ trivially holds. Thus, again making use of Theorem 1 we find that

$$
\phi(X) \leq_{\mathrm{ttt}-2} \phi(Y) .
$$

Clearly, the function $\phi(\cdot)$ is neither concave nor convex on $\Re_{+}$. Hence, the condition " $\phi(\cdot)$ is concave" in Theorem 11 is only a sufficient condition, but not necessary.

Theorem 12. Let $\phi(\cdot)$ be a non-negative and increasing convex function defined on $\Re_{+}=[0, \infty)$ such that $\phi(0)=0$. If $\phi(X) \leq_{t t t-n} \phi(Y)$, then $X \leq_{t t t-n} Y$.

Remark 11. In Theorem 12, the condition " $\phi(\cdot)$ is convex" is only a sufficient condition, but not necessary. The above Counterexample 6 proves this assertion.

\section{Preservation of the TTT- $n$ Ordering in Several Stochastic Models}

Marshall and Olkin [32], Sankaran and Jayakumar [33] and Navarro et al. [34] studied the following proportional odds models. Let $X$ be a non-negative continuous random variable with the distribution function $F_{X}$ and density function $f_{X}$. The proportional odds random variable, denoted by $X_{p}$, is defined by the distribution function

$$
\bar{F}_{X_{\mathrm{p}}}(x)=\frac{\theta \bar{F}_{X}(x)}{1-(1-\theta) \bar{F}_{X}(x)}
$$

for $\theta>0$, where $\theta$ is a proportional constant. Let $Y$ be another non-negative continuous random variable with distribution function $G_{Y}$ and density function $g_{Y}$. Similarly, define the proportional odds random variable $Y_{p}$ of $Y$ by the distribution function

$$
\bar{G}_{Y_{\mathrm{p}}}(x)=\frac{\theta \bar{G}_{Y}(x)}{1-(1-\theta) \bar{G}_{Y}(x)}
$$


for $\theta>0$, where the proportional constant $\theta$ is the same as above.

For the proportional odds models, we obtain the following result.

Theorem 13. Let $X, Y, X_{p}$ and $Y_{p}$ be as described above.

(a) Assume $0<\theta<1$. If $X \leq_{t t t-n} Y$, then $X_{p} \leq_{t t t-n} Y_{p}$.

(b) Assume $\theta>1$. If $X_{p} \leq_{t t t-n} Y_{p}$, then $X \leq_{t t t-n} Y$.

Proof. We only give the proof for (a), the proof of $(b)$ is similar and hence is omitted here. Denote the function

$$
h(u)=\frac{\theta u}{1-(1-\theta) u}, \quad u \in[0,1],
$$

for any $\theta>0$. It is easy to see that

(i) If $0<\theta<1$, then $h(u)$ is increasing convex on $[0,1]$.

(ii) If $\theta>1$, then $h(u)$ is increasing concave on $[0,1]$.

From the definition of $X_{p}$ and $Y_{p}$, we have

$$
\bar{F}_{X_{\mathrm{p}}}(x)=h\left[\bar{F}_{X}(x)\right] \text { and } \bar{G}_{Y_{\mathrm{p}}}(x)=h\left[\bar{G}_{Y}(x)\right], \quad \text { for all } x \geq 0 .
$$

Hence, the density functions of $X_{p}$ and $Y_{p}$ are, respectively,

$$
f_{X_{\mathrm{p}}}(x)=h^{\prime}\left[\bar{F}_{X}(x)\right] f_{X}(x) \quad \text { and } \quad g Y_{\mathrm{p}}(x)=h^{\prime}\left[\bar{G}_{Y}(x)\right] g_{Y}(x), \quad \text { for all } x \geq 0 .
$$

It can be proven that $G_{Y_{\mathrm{p}}}^{-1}\left[F_{X_{\mathrm{p}}}(x)\right]=G_{Y}^{-1}\left[F_{X}(x)\right]$, by differentiating this equation we have

$$
\frac{f_{X_{\mathrm{p}}}(x)}{g_{Y_{\mathrm{p}}}\left[G_{Y_{\mathrm{p}}}^{-1}\left(F_{X_{\mathrm{p}}}(x)\right)\right]}=\frac{f_{X}(x)}{g_{Y}\left[G_{Y}^{-1}\left(F_{X}(x)\right)\right]} .
$$

If $X \leq_{\mathrm{ttt}-n} Y$, from Theorem 1 , we find that

$$
\int_{0}^{t}\left[\bar{F}_{X}(x)\right]^{n+1}\left[\frac{f_{X}(x)}{g_{Y}\left[G_{Y}^{-1}\left(F_{X}(x)\right)\right]}-1\right] \mathrm{d} x \geq 0, \quad \text { for all } t \geq 0 .
$$

Since $h(u)$ is increasing convex on $[0,1]$ when $0<\theta \leq 1$, hence $h(u) / u$ is non-negative and increasing in $x$. Thus, we find that the function

$$
\left[h\left[\bar{F}_{X}(x)\right] / \bar{F}_{X}(x)\right]^{n+1} \quad \text { is non-negative and decreasing. }
$$

Making use of (53)-(55) and Lemma 1, we have

$$
\int_{0}^{t}\left[h\left[\bar{F}_{X}(x)\right]\right]^{n+1}\left[\frac{f_{X_{\mathrm{p}}}(x)}{g_{Y_{\mathrm{p}}}\left[G_{Y_{\mathrm{p}}}^{-1}\left(F_{X_{\mathrm{p}}}(x)\right)\right]}-1\right] \mathrm{d} x \geq 0, \quad \text { for all } t \geq 0,
$$

again, by Theorem 1 in turn, which states that

$$
X_{\mathrm{p}} \leq_{\mathrm{ttt}-n} Y_{\mathrm{p}}
$$

This completes the proof.

Remark 12. Theorem 13 (a) says that the TTT-n ordering is closed with respect to the proportional odds model when the proportional constant $0<\theta<1$. Theorem $13(b)$ says that the TTT-n ordering has the reversed closure property with respect to the proportional odds model when the proportional constant $\theta>1$. 
In the following, we investigate the preservation of the TTT- $n$ ordering in a record values model. Chandler [35] introduced and studied some basic properties of records. Furthermore, much progress on stochastic orderings of record values refer to Khaledi et al. [36], Kundu et al. [37], Zhao and Balakrishnan [38], Zarezadeh and Asadi [39], Li and Zhang [40], Kang [41-43], Kang and Yan [44], Yan [45], and the references therein.

Let $\left\{X_{i}, i \geq 1\right\}$ be a sequence of independent and identically distributed random variables from an absolutely continuous non-negative random variable $X$, where $X$ has its probability density function $f_{X}$ and the survival function $\bar{F}_{X}$, and let $k$ be a positive integer. The random variables $T_{k}^{X}(m)$, defined recursively by $T_{k}^{X}(1)=1$ and

$$
T_{k}^{X}(m+1)=\min \left\{j>T_{k}^{X}(m): X_{j: j+k-1}>X_{T_{k}^{X}(m): T_{k}^{X}(m)+k-1}\right\}, \quad m \geq 1,
$$

are called the $m$ th $k$-record times, and the quantities $X_{T_{k}^{X}(m): T_{k}^{X}(m)+k-1}$, written as $R_{m, k^{\prime}}^{X}$ are called the $m$ th $k$-record values. For $k=1$, the $k$-record values model reduces to the well-known ordinary record values model, and $T_{k}^{X}(m)$ and $R_{m, k}^{X}$ are abbreviated to $T^{X}(m)$ and $R_{m}^{X}$ for all $m \geq 1$. For more details, readers can refer to Ahsanullah [46] and Arnold et al. [47].

The sequence of $k$-record values was introduced by Dziubdziela and Kopociński [48] through observing successive $k$ th largest values in a sequence. They actually called them $k$ th record values. It is easy to prove that the probability density function and the survival function of $R_{m, k}^{X}$ can be expressed as, respectively,

$$
f_{R_{m, k}^{X}}(x)=\frac{k^{m}}{\Gamma(m)} \Lambda_{X}^{m-1}(x) \bar{F}_{X}^{k-1}(x) f_{X}(x),
$$

and

$$
\bar{F}_{R_{m, k}^{X}}(x)=\bar{F}_{X}^{k}(x) \sum_{j=0}^{m-1} \frac{\left(k \Lambda_{X}(x)\right)^{j}}{j !}=\bar{\Gamma}_{m}\left(k \Lambda_{X}(x)\right),
$$

for all $x \geq 0$, where $\bar{\Gamma}_{m}(\cdot)$ is the survival function of a gamma random variable with a shape parameter $m$ and a scale parameter $\lambda>0$, and $\Lambda_{X}(x)=-\ln \bar{F}_{X}(x)$ is the cumulative hazard rate function of $X$.

Let $X$ and $Y$ be two non-negative random variable with the survival functions $\bar{F}_{X}(x)$ and $\bar{G}_{Y}(x)$, the probability density functions $f_{X}(x)$ and $g_{Y}(x)$, and the hazard rate functions $\lambda_{X}(x)$ and $\lambda_{Y}(x)$, respectively. The following stochastic orderings are useful in the proof of the following theorem (see Shaked and Shanthikumar [18]).

- $\quad X$ is said to be smaller than $Y$ in the likelihood ratio order if $g_{Y}(x) / f_{X}(x)$ is increasing in $X$, denoted by $X \leq_{\operatorname{lr}} Y$.

- $\quad X$ is said to be smaller than $Y$ in the hazard rate order if $\lambda_{X}(x) \geq \lambda_{Y}(x)$ for all $x$, equivalently, if $\bar{G}_{Y}(x) / \bar{F}_{X}(x)$ is increasing in $x$, denoted by $X \leq_{\mathrm{hr}} Y$.

It is well known (also see Shaked and Shanthikumar [18]) that

$$
X \leq_{\mathrm{lr}} Y \Longrightarrow X \leq_{\mathrm{hr}} Y .
$$

By using (56), we easily find that the function

$$
\frac{f_{R_{m, k}^{X}}(x)}{f_{R_{m, l}^{X}}(x)}=\left(\frac{k}{l}\right)^{m}\left[\bar{F}_{X}(x)\right]^{k-l}
$$

is increasing in $x \geq 0$ when $l>k \geq 1$. From the above definition of the likelihood ratio order, we immediately get that

$$
R_{m, l}^{X} \leq_{\operatorname{lr}} R_{m, k}^{X} \quad \text { whenever } l>k \geq 1 .
$$


We easily obtain the following lemma.

Lemma 4. The function $\bar{F}_{R_{m, l}^{X}}(x) / \bar{F}_{R_{m, k}^{X}}(x)$ is decreasing in $x \geq 0$ whenever $l>k \geq 1$.

Proof. The proof is immediate by using (58), (60), and the above definition of the hazard rate order.

Now we consider the preservation of the TTT- $n$ ordering in the record values model.

Theorem 14. Let $X$ and $Y$ be two absolutely continuous and non-negative random variables, $j, k, l, m$, and $n$ be positive integers. Then,

(a) $R_{m, k}^{X} \leq_{t t t-n} R_{m, k}^{Y} \Longrightarrow R_{m, l}^{X} \leq_{t t t-n} R_{m, l^{\prime}}^{Y}$ for all $m \geq 1, l>k \geq 1$.

Particularly, $R_{m, k-1}^{X} \leq_{t t t-n} R_{m, k-1}^{Y} \Longrightarrow R_{m, k}^{X} \leq_{t t t-n} R_{m, k^{\prime}}^{Y} \quad$ for all $m \geq 1, k \geq 2$.

(b) $R_{m, k}^{X} \leq_{t t t-n} R_{m, k}^{Y} \Longrightarrow R_{j, k}^{X} \leq_{t t t-n} R_{j, k^{\prime}}^{Y}$ for all $m>j \geq 1, k \geq 1$.

Particularly, $R_{m, k}^{X} \leq_{t t t-n} R_{m, k}^{Y} \Longrightarrow R_{m-1, k}^{X} \leq_{t t t-n} R_{m-1, k^{\prime}}^{Y} \quad$ for all $m \geq 2, k \geq 1$.

(c) $R_{m, k}^{X} \leq_{t t t-n} R_{m, k}^{Y, k} \Longrightarrow R_{j, l}^{X} \leq_{t t t-n} R_{j, l}^{Y} \quad$ for all $m>j \geq 1, l>k \geq 1$.

Particularly, $R_{m+1, k-1}^{X} \leq_{t t t-n} R_{m+1, k-1}^{Y} \Longrightarrow R_{m, k}^{X} \leq_{t t t-n} R_{m, k^{\prime}}^{Y}$ for all $m \geq 1, k \geq 2$.

Proof. (a) Suppose that $R_{m, k}^{X} \leq_{\mathrm{ttt}-n} R_{m, k}^{Y}$. Then, from Theorem 1, we find that

$$
\int_{0}^{t}\left[\bar{F}_{R_{m, k}^{X}}(x)\right]^{n+1}\left[\frac{f_{R_{m, k}^{X}}(x)}{g_{R_{m, k}^{Y}}\left[G_{R_{m, k}^{Y}}^{-1}\left(F_{R_{m, k}^{X}}(x)\right)\right]}-1\right] \mathrm{d} x \geq 0, \quad \text { for all } t \geq 0 .
$$

By using (56) and (57) and noticing that

$$
\bar{G}_{R_{m, k}^{Y}}^{-1}\left(\bar{F}_{R_{m, k}^{X}}(x)\right)=G_{R_{m, k}^{Y}}^{-1}\left(F_{R_{m, k}^{X}}(x)\right) \text { and } \bar{G}_{R_{m, l}^{Y}}^{-1}\left(\bar{F}_{R_{m, l}^{X}}(x)\right)=G_{R_{m, l}^{Y}}^{-1}\left(F_{R_{m, l}^{X}}(x)\right) \text {, }
$$

one can prove that, for all positive integers $m, l, k$ and real $x \geq 0$,

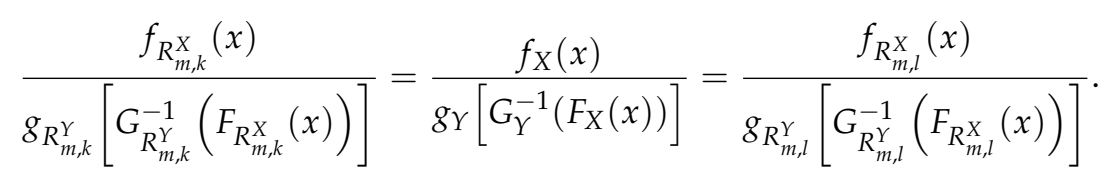

Furthermore, by Theorem 1 , we see that $R_{m, l}^{X} \leq_{\mathrm{ttt}-n} R_{m, l}^{Y}$ if and only if the inequality

$$
\int_{0}^{t}\left[\bar{F}_{R_{m, l}^{X}}(x)\right]^{n+1}\left[\frac{f_{R_{m, l}^{X}}(x)}{g_{R_{m, l}^{Y}\left[G_{R_{m, l}^{Y}}^{Y}\left(F_{R_{m, l}^{X}}(x)\right)\right]}}-1\right] \mathrm{d} x \geq 0, \quad \text { for all } t \geq 0 .
$$

Making use of (61), together with (62), Lemmas 1 and 4, we find that inequality (63) is valid at once. This completes the proof of part (a).

(b) By means of a similar method of part (a), the required result of part (b) follows.

(c) By using the results of parts (a) and (b) simultaneously, the desired result follows immediately.

The following theorem deals with the preservation of the TTT- $n$ ordering in a proportional reversed hazard rate model. For more details about this model, one may refer to Gupta and Gupta [49], and Di Crescenzo and Longobardi [50].

Let $X$ and $Y$ be two absolutely continuous non-negative random variables with respective distribution functions $F_{X}$ and $G_{Y}$. For a real constant $\theta>0$, let $X(\theta)$ and $Y(\theta)$ denote another two random variables with respective distribution functions $\left(F_{X}\right)^{\theta}$ and 
$\left(G_{Y}\right)^{\theta}$. Suppose that $X$ and $Y$ have 0 as the common left endpoint of their supports. Then, we have the following results.

Theorem 15. Let $X, Y, X(\theta)$ and $Y(\theta)$ be as described above.

(a) Assume that $0<\theta<1$. If $X \leq_{t t t-n} Y$, then $X(\theta) \leq_{t t t-n} Y(\theta)$.

(b) Assume that $\theta>1$. If $X(\theta) \leq_{t t t-n} Y(\theta)$, then $X \leq_{t t t-n} Y$.

Proof. Assume that $X, Y, X(\theta)$ and $Y(\theta)$ have respective distribution functions $\bar{F}_{X}, \bar{G}_{Y}$, $\bar{F}_{X(\theta)}$ and $\bar{G}_{Y(\theta)}$, the density functions $f_{X}, g_{Y}, f_{X(\theta)}$ and $g_{Y(\theta)}$, and the quantile functions $F_{X}^{-1}, G_{Y}^{-1}, F_{X(\theta)}^{-1}$ and $G_{Y(\theta)}^{-1}$, respectively.

(a) Suppose that $X \leq_{\mathrm{ttt}-n} Y$. Then, from Theorem 1 we have

$$
\int_{0}^{t}\left[\bar{F}_{X}(x)\right]^{n+1}\left[\frac{f_{X}(x)}{g_{Y}\left[G_{Y}^{-1}\left(F_{X}(x)\right)\right]}-1\right] \mathrm{d} x \geq 0, \quad \text { for all } t \geq 0 .
$$

From the definition of the above proportional reversed hazard rate model, we find that the distribution functions of $X(\theta)$ and $Y(\theta)$ are given, respectively, by

$$
F_{X(\theta)}(x)=\left[F_{X}(x)\right]^{\theta}, \quad G_{Y(\theta)}(x)=\left[G_{Y}(x)\right]^{\theta}, \quad x \geq 0,
$$

By using (65) we find that

$$
G_{Y(\theta)}^{-1}\left[F_{X(\theta)}(x)\right]=G_{Y}^{-1}\left[F_{X}(x)\right], \quad x \geq 0 .
$$

Differentiating (66), we have

$$
\frac{f_{X(\theta)}(x)}{g_{Y(\theta)}\left[G_{Y(\theta)}^{-1}\left(F_{X(\theta)}(x)\right)\right]}=\frac{f_{X}(x)}{g_{Y}\left[G_{Y}^{-1}\left(F_{X}(x)\right)\right]} .
$$

Moreover, it is easy to see that the density function of $X(\theta)$ is given by

$$
f_{X(\theta)}(x)=\theta f_{X}(x)\left[F_{X}(x)\right]^{\theta-1},
$$

then, $\frac{f_{X(\theta)}(x)}{f_{X}(x)}=\theta\left[F_{X}(x)\right]^{\theta-1}$. Thus,

$$
\frac{\mathrm{d}}{\mathrm{d} x}\left(\frac{f_{X(\theta)}(x)}{f_{X}(x)}\right)=\theta(\theta-1) f_{X}(x)\left[F_{X}(x)\right]^{\theta-2}, \quad x \geq 0 .
$$

Hence, we find that:

(i) The function $\frac{f_{X(\theta)}(x)}{f_{X}(x)}$ is decreasing in $x$ when $0<\theta<1$.

(ii) The function $\frac{f_{X}(x)}{f_{X(\theta)}(x)}$ is decreasing in $x$ when $\theta>1$.

By using the definitions of the orders $\leq_{\mathrm{lr}}$ and $\leq_{\mathrm{hr}}$ and using the implication relation (58), we find that:

(iii) The function $\frac{\bar{F}_{X(\theta)}(x)}{\bar{F}_{X}(x)}$ is decreasing in $x$ when $0<\theta<1$.

(iv) The function $\frac{\bar{F}_{X}(x)}{\bar{F}_{X(\theta)}(x)}$ is decreasing in $x$ when $\theta>1$.

Making use of this fact (iii), together with (64), (67) and Lemma 1 we find that

$$
\int_{0}^{t}\left[\bar{F}_{X(\theta)}(x)\right]^{n+1}\left[\frac{f_{X(\theta)}(x)}{g_{Y(\theta)}\left[G_{Y(\theta)}^{-1}\left(F_{X(\theta)}(x)\right)\right]}-1\right] \mathrm{d} x \geq 0, \quad \text { for all } t \geq 0,
$$


which, in turn by Theorem 1 , asserts that $X(\theta) \leq_{\mathrm{ttt}-n} Y(\theta)$. This proves (a).

(b) The proof is similar to that of (a). Thus, the proof is complete.

Remark 13. Theorem 15 (a) says that the TTT-n ordering is closed with respect to the proportional reversed hazard rate model when the proportional constant $0<\theta<1$. Theorem 15 (b) says that the TTT-n ordering has the reversed closure property with respect to the proportional reversed hazard rate model when the proportional constant $\theta>1$.

Remark 14. In Theorem $15(b)$, when the proportional constant $\theta>1$ takes a natural number $m \geq 1$, then, Theorem 16 (b) becomes Theorem 7 . Hence, Theorem 7 is a special case of Theorem 16 (b).

Then, we consider the preservation of the TTT- $n$ ordering in a proportional hazard rate model. For more details on the proportional hazard rate model, we refer to Nanda and Paul [51], Gupta and Gupta [49], Di Crescenzo and Longobardi [50], Abbasnejad et al. [52], and Shaked and Shanthikumar [18].

Let $X$ and $Y$ be two absolutely continuous non-negative random variables random variables with survival functions $\bar{F}_{X}$ and $\bar{G}_{Y}$, respectively. For a positive real constant $\theta>0$, let $X(\theta)$ and $Y(\theta)$ denote another two non-negative random variables with survival functions $\left[\bar{F}_{X}(x)\right]^{\theta}$ and $\left[\bar{G}_{Y}(x)\right]^{\theta}$, respectively. Suppose that $X$ and $Y$ have 0 as the common left endpoint of their supports. Nair et al. ([26], p. 1137, Theorem 5.2) considered the preservation of the TTT- $n$ ordering in this proportional hazard rate model. They obtained the following results. For the convenience of citation, we list these results here.

Theorem 16 (Nair et al. [26]). Let $X, Y, X(\theta)$ and $Y(\theta)$ be as described above.

(a) Assume that $\theta>1$. If $X \leq_{t t t-n} Y$, then $X(\theta) \leq_{t t t-n} Y(\theta)$.

(b) Assume that $0<\theta<1$. If $X(\theta) \leq_{t t t-n} Y(\theta)$, then $X \leq_{t t t-n} Y$.

Remark 15. Theorem 16 (a) says that the TTT-n ordering is closed with respect to the proportional hazard rate model when the proportional constant $\theta>1$. Theorem 16 (b) says that the TTT- $n$ ordering has the reversed closure property with respect to the proportional hazard rate model when the proportional constant $0<\theta<1$.

Remark 16. In Theorem 16 (a), when the proportional constant $\theta>1$ takes a natural number $m \geq 1$, then, Theorem 16 (a) becomes Theorem 8.

In the following, we investigate the preservations of the TTT- $n$ ordering in the mixture model of proportional hazard rate which can be viewed as a generalization of Theorem 5.2 in Nair et al. [26].

Let $X$ and $Y$ be two absolutely continuous non-negative random variables random variables with survival functions $\bar{F}_{X}$ and $\bar{G}_{Y}$, respectively. For a positive-valued random variable $\Theta$, let $X(\Theta)$ and $Y(\Theta)$ denote another two non-negative random variables with survival functions $\mathbb{E}\left[\left(\bar{F}_{X}(x)\right)^{\Theta}\right]$ and $\mathbb{E}\left[\left(\bar{G}_{Y}(x)\right)^{\Theta}\right]$, respectively. Suppose that $X$ and $Y$ have 0 as the common left endpoint of their supports. For this mixture proportional hazard rate model we obtain the following results.

Theorem 17. Let $X, Y, X(\Theta)$, and $Y(\Theta)$ be as described above.

(a) Assume that $\Theta>1$ almost surely. If $X \leq_{t t t-n} Y$, then $X(\Theta) \leq_{t t t-n} Y(\Theta)$.

(b) Assume that $0<\Theta<1$ almost surely. If $X(\Theta) \leq_{t t t-n} Y(\Theta)$, then $X \leq_{t t t-n} Y$.

Proof. Assume that $X, Y, X(\Theta)$ and $Y(\Theta)$ have respective survival functions $\bar{F}_{X}, \bar{G}_{Y}, \bar{F}_{X(\Theta)}$ and $\bar{G}_{Y(\Theta)}$, the density functions $f_{X}, g_{Y}, f_{X(\Theta)}$ and $g_{Y(\Theta)}$, and the quantile functions $F_{X}^{-1}$, $G_{Y}^{-1}, F_{X(\Theta)}^{-1}$ and $G_{Y(\Theta)}^{-1}$, respectively. 
(a) Suppose that $X \leq_{\mathrm{ttt}-n} Y$. Then, from Theorem 1, we have

$$
\int_{0}^{t}\left[\bar{F}_{X}(x)\right]^{n+1}\left[\frac{f_{X}(x)}{g_{Y}\left[G_{Y}^{-1}\left(F_{X}(x)\right)\right]}-1\right] \mathrm{d} x \geq 0, \quad \text { for all } t \geq 0 .
$$

In view of the above definition of the mixture model of proportional hazard rate, we find that the survival functions of $X(\Theta)$ and $Y(\Theta)$ are given, respectively, by

$$
\bar{F}_{X(\Theta)}(x)=\mathbb{E}\left[\left(\bar{F}_{X}(x)\right)^{\Theta}\right]=L_{\Theta}\left(\Lambda_{X}(x)\right), \quad \bar{G}_{Y(\Theta)}(x)=\mathbb{E}\left[\left(\bar{G}_{Y}(x)\right)^{\Theta}\right]=L_{\Theta}\left(\Lambda_{Y}(x)\right), \quad x \geq 0,
$$

where $L_{\Theta}(\cdot)$ is the Laplace transform of $\Theta, \Lambda_{X}(\cdot)$ and $\Lambda_{Y}(\cdot)$ are the cumulative hazard rate functions of $X$ and $Y$, respectively. By using (69) we get that

$$
\bar{G}_{Y(\Theta)}^{-1}\left[\bar{F}_{X(\Theta)}(x)\right]=G_{Y(\Theta)}^{-1}\left[F_{X(\Theta)}(x)\right]=\Lambda_{X}^{-1}\left[\Lambda_{Y}(x)\right]=G_{Y}^{-1}\left[F_{X}(x)\right], \quad x \geq 0 .
$$

That is,

$$
G_{Y(\Theta)}^{-1}\left[F_{X(\Theta)}(x)\right]=G_{Y}^{-1}\left[F_{X}(x)\right], \quad x \geq 0 .
$$

Differentiating (70) we have

$$
\frac{f_{X(\Theta)}(x)}{g_{Y(\Theta)}\left[G_{Y(\Theta)}^{-1}\left(F_{X(\Theta)}(x)\right)\right]}=\frac{f_{X}(x)}{g_{Y}\left[G_{Y}^{-1}\left(F_{X}(x)\right)\right]} .
$$

Furthermore, also from (69) we have

$$
\frac{\bar{F}_{X(\Theta)}(x)}{\bar{F}_{X}(x)}=\mathbb{E}\left[\left(\bar{F}_{X}(x)\right)^{\Theta-1}\right], \quad x \geq 0 .
$$

Thus,

$$
\frac{\mathrm{d}}{\mathrm{d} x}\left(\frac{\bar{F}_{X(\Theta)}(x)}{\bar{F}_{X}(x)}\right)=-f_{X}(x) \mathbb{E}\left[(\Theta-1)\left(\bar{F}_{X}(x)\right)^{\Theta-2}\right] \leq 0, \quad x \geq 0 .
$$

Hence, the function $\frac{\bar{F}_{X(\Theta)}(x)}{\bar{F}_{X}(x)}$ is decreasing in $x \geq 0$ when $\Theta>1$ almost surely and is increasing in $x \geq 0$ when $0<\Theta<1$ almost surely. Making use of this fact, together with (68), (71) and Lemma 1, we find that

$$
\int_{0}^{t}\left[\bar{F}_{X(\Theta)}(x)\right]^{n+1}\left[\frac{f_{X(\Theta)}(x)}{g_{Y(\Theta)}\left[G_{Y(\Theta)}^{-1}\left(F_{X(\theta)}(x)\right)\right]}-1\right] \mathrm{d} x \geq 0, \quad \text { for all } t \geq 0,
$$

which, by Theorem 1 in turn, asserts that $X(\Theta) \leq_{\mathrm{ttt}-n} Y(\Theta)$. This proves (a).

(b) The proof is similar to that of (a). Thus, the proof is complete.

Remark 17. Theorem 17 (a) says that the TTT-n ordering is closed with respect to the mixture proportional hazard rate model under the condition of the proportional random variable $\Theta>1$ almost surely. Theorem 17 (b) says that the TTT-n ordering has the reversed closure property with respect to the mixture proportional hazard rate model under the condition of the proportional random variable $0<\Theta<1$ almost surely.

Remark 18. In Theorem 17, when the proportional random variable $\Theta$ is degenerated as a positive constant $\theta$, then, Theorem 17 becomes Theorem 5.2 in Nair et al. [26]. Furthermore, in Theorem $17(a)$, when the proportional random variable $\Theta$ is degenerated as a natural number $m \geq 1$, then Theorem 17 (a) becomes Theorem 8. Hence, Theorem 8 is a special case of Theorem 17 (a). 
We now deal with the preservation of the TTT- $n$ ordering in the mixture proportional reversed hazard rate model.

Let $X$ and $Y$ be two absolutely continuous non-negative random variables with distribution functions $F_{X}$ and $G_{Y}$, respectively. For a positive-valued random variable $\Theta$, let $X(\Theta)$ and $Y(\Theta)$ denote another two non-negative random variables with survival functions $\mathbb{E}\left[\left(F_{X}(x)\right)^{\Theta}\right]$ and $\mathbb{E}\left[\left(G_{Y}(x)\right)^{\Theta}\right]$, respectively. Suppose that $X$ and $Y$ have 0 as the common left endpoint of their supports. Then, we have the following results. The proofs are similar to that of Theorem 15 and the details are omitted here.

Theorem 18. Let $X, Y, X(\Theta)$, and $Y(\Theta)$ be as described above.

(a) Assume that $0<\Theta<1$ almost surely. If $X \leq_{t t t-n} Y$, then $X(\Theta) \leq_{t t t-n} Y(\Theta)$.

(b) Assume that $\Theta>1$ almost surely. If $X(\Theta) \leq_{t t t-n} Y(\Theta)$, then $X \leq_{t t t-n} Y$.

Remark 19. Theorem 18 (a) says that the TTT-n ordering is closed with respect to the mixture proportional reversed hazard rate model under the condition of the proportional random variable $0<\Theta<1$ almost surely. Theorem 18 (b) says that the TTT- $n$ ordering has the reversed closure with respect to the mixture proportional reversed hazard rate model under the condition of the proportional random variable $\Theta>1$ almost surely.

Remark 20. In Theorem $18(b)$, when the proportional random variable $\Theta$ is degenerated as $a$ natural number $m>1$, then, Theorem $18(b)$ becomes Theorem 7 . Hence, Theorem 7 is a special case of Theorem $18(b)$.

\section{Closure and Reversed Closure Properties of the TTT- $n$ Ordering for Coherent Systems with Dependent and Identically Distributed Components}

Navarro et al. [53] gave a convenient representation of a coherent system reliability $\bar{F}_{T}$. They proved the following result. For the ease of citation, we give this result as a lemma.

Lemma 5 (Navarro et al. [53]). Let $T=\phi\left(X_{1}, \ldots, X_{n}\right)$ be the lifetime of a coherent system based on possibly dependent components with lifetimes $X_{1}, \ldots, X_{n}$, having a common reliability function $\bar{F}_{X}(t)=\operatorname{Pr}\left(X_{i}>t\right)$. Assume that $h$ is a distortion function. Then, the system reliability function can be written as

$$
\bar{F}_{T}(t)=h\left(\bar{F}_{X}(t)\right),
$$

where $h$ only depends on $\phi$ and on the survival copula of $\left(X_{1}, \ldots, X_{n}\right)$.

Making use of (72), the distribution function of the coherent system lifetime $T$ is given by

$$
F_{T}(t)=1-\bar{F}_{T}(t)=1-h\left(1-F_{X}(t)\right)=g\left(F_{X}(t)\right),
$$

where $g(u)=1-h(1-u), u \in(0,1)$. Then, we obtain the following result.

Theorem 19. Let $X$ and $Y$ be two non-negative continuous random variables with survival functions $\bar{F}_{X}(t)$ and $\bar{G}_{Y}(t)$, respectively. Let $T_{1}=\phi\left(X_{1}, \ldots, X_{n}\right)$ and $T_{2}=\phi\left(Y_{1}, \ldots, Y_{n}\right)$ be the lifetimes of two coherent systems with common structure function $\phi$ and with identically distributed component lifetimes $X_{1}, \ldots, X_{n}$ and $Y_{1}, \ldots, Y_{n}$, having common continuous survival functions $\bar{F}_{X}(t)=\operatorname{Pr}\left(X_{i}>t\right)$ and $\bar{G}_{Y}(t)=\operatorname{Pr}\left(Y_{i}>t\right)$ for $i=1, \ldots, n$, respectively. Let $h$ be the common domination function of these two coherent systems.

(a) Assume $h(x) / x$ is increasing in $x>0$. If $X \leq_{t t t-n} Y$, then $T_{1} \leq_{t t t-n} T_{2}$.

(b) Assume $h(x) / x$ is decreasing in $x>0$. If $T_{1} \leq_{t t t-n} T_{2}$, then $X \leq_{t t t-n} Y$.

Proof. Suppose that $T_{1}$ and $T_{2}$ have survival functions $\bar{F}_{T_{1}}(x)$ and $\bar{G}_{T_{2}}(x)$, distribution functions $F_{T_{1}}(x)$ and $G_{T_{2}}(x)$, density functions $f_{T_{1}}(x)$ and $g_{T_{2}}(x)$, and quantile functions $F_{T_{1}}^{-1}(x)$ and $G_{T_{2}}^{-1}(x)$, respectively. 
By (72), it can be proven that $G_{T_{2}}^{-1}\left[F_{T_{1}}(x)\right]=\bar{G}_{T_{2}}^{-1}\left[\bar{F}_{T_{1}}(x)\right]=G_{Y}^{-1}\left[F_{X}(x)\right]$, by differentiating this equation, we obtain

$$
\frac{f_{T_{1}}(x)}{g_{T_{2}}\left[G_{T_{2}}^{-1}\left(F_{T_{1}}(x)\right)\right]}=\frac{f_{X}(x)}{g_{Y}\left[G_{Y}^{-1}\left(F_{X}(x)\right)\right]}, \text { for all } x>0 .
$$

In view of Theorem 1 , we find that $X \leq_{\mathrm{ttt}-n} Y$ if, and only if,

$$
\int_{0}^{t}\left[\bar{F}_{X}(x)\right]^{n+1}\left[\frac{f_{X}(x)}{g_{Y}\left[G_{Y}^{-1}\left(F_{X}(x)\right)\right]}-1\right] \mathrm{d} x \geq 0, \quad \text { for all } t \geq 0 ;
$$

Furthermore, that $T_{1} \leq_{\mathrm{LQE}} T_{2}$ if, and only if,

$$
\int_{0}^{t}\left[\bar{F}_{T_{1}}(x)\right]^{n+1}\left[\frac{f_{T_{1}}(x)}{g_{T_{2}}\left[G_{T_{2}}^{-1}\left(F_{T_{1}}(x)\right)\right]}-1\right] \mathrm{d} x \geq 0, \quad \text { for all } t \geq 0,
$$

or, by using (72), equivalently,

$$
\int_{0}^{t}\left(h\left[\bar{F}_{X}(x)\right]\right)^{n+1}\left[\frac{f_{X}(x)}{g_{Y}\left[G_{Y}^{-1}\left(F_{X}(x)\right)\right]}-1\right] \mathrm{d} x \geq 0, \quad \text { for all } t \geq 0 .
$$

(a) Assume that $X \leq_{\mathrm{ttt}-n} Y$. If $h(x) / x$ is increasing in $x>0$, then, the function

$$
h\left[\bar{F}_{X}(x)\right] / \bar{F}_{X}(x)
$$

is non-negative and decreasing in $x>0$. By Lemma 1, (73) and (74), we see that the inequality (76) holds, which asserts by Theorem 1 that $T_{1} \leq_{\mathrm{ttt}-n} T_{2}$.

(b) Assume that $T_{1} \leq_{\mathrm{ttt}-n} T_{2}$. If $h(x) / x$ is decreasing in $x>0$, then, the function

$$
\bar{F}_{X}(x) / h\left[\bar{F}_{X}(x)\right]
$$

is non-negative and decreasing in $x>0$. By Lemma 1 and (76), we see that the inequality (74) holds, which asserts by Theorem 1 that $X \leq_{\mathrm{ttt}-n} Y$.

Remark 21. In Theorem 19, if $X_{1}, \ldots, X_{n}$ and $Y_{1}, \ldots, Y_{n}$ are i.i.d., respectively, then, Theorem 19 (a) becomes as Theorems 8 and 19 (b) becomes as Theorem 7 . Hence Theorems 8 and 7 are special cases of Theorem 19.

Similarly, if $X_{1}, X_{2}, \ldots$ and $Y_{1}, Y_{2}, \ldots$ are i.i.d., respectively, then, Theorem 19 (a) becomes as Theorems 9 and 19 (b) becomes as Theorem 10. Hence Theorems 9 and 10 are also special cases of Theorem 19.

\section{Conclusions}

The concept of TTT transform is of great significance in engineering and technologies, experiment science, and other related scientific research fields. The TTT ordering compares two random lifetimes, hence the TTT ordering is also of important meanings. It takes a central position, and plays a key role in the stochastic order theory and in the reliability theory. Similarly, the TTT- $n$ ordering is also of the same functions and positions in stochastic order theory as the TTT ordering is. Hence, we focus our attention on the research of the TTT- $n$ ordering. 
In this paper, we examine some characterizations of the TTT- $n$ ordering. Firstly, we obtain the closure and reversed closure properties of this new stochastic order under several reliability operations. Secondly, we investigate preservation results of this order in several stochastic models. Finally, we also consider the closure and reversed closure properties of the TTT- $n$ ordering for coherent systems with dependent and identically distributed components. We get that the TTT- $n$ ordering is:

(1) Closed respect to a series system; but not closed respect to a parallel system. This case can be viewed as a kind of anti-symmetry.

(2) Closed respect to a random series system; but not closed respect to a random parallel system. This case can be viewed as a kind of anti-symmetry.

(3) Reversely closed respect to a parallel system; but not reversely closed respect to a series system. This case can be viewed as a kind of anti-symmetry.

(4) Reversely closed respect to a random parallel system; but not reversely closed respect to a random series system. This case can be viewed as a kind of anti-symmetry.

(5) Closed under a non-negative, increasing and concave transform; but not closed under a non-negative, increasing and convex transform. This case can be viewed as a kind of anti-symmetry.

(6) Reversely closed under a non-negative, increasing and convex transform; but not closed under a non-negative, increasing and concave transform. This case can be viewed as a kind of anti-symmetry.

(7) Closed but not reversely closed under some appropriate conditions in the proportional odds models. This case can be viewed as a kind of anti-symmetry.

(8) Reversely closed but not closed under other appropriate conditions in the proportional odds models. This case can be viewed as a kind of anti-symmetry.

(9) Closed but not reversely closed under some appropriate conditions in the proportional reversed hazard rate models. This case can be viewed as a kind of anti-symmetry.

(10) Reversely closed but not closed under other appropriate conditions in the proportional reversed hazard rate models. This case can be viewed as a kind of anti-symmetry.

(11) Closed but not reversely closed under some appropriate conditions in the proportional hazard rate and mixture proportional hazard rate models. These cases can be viewed as a kind of anti-symmetry.

(12) Reversely closed but not closed under other appropriate conditions in the proportional hazard rate and mixture proportional hazard rate models. These cases can be viewed as a kind of anti-symmetry.

(13) Closed and reversely closed, respectively, under some appropriate conditions in the record-value models; the cases are more complex. This case can be viewed as a kind of anti-symmetry.

(14) Closed but not reversely closed under some appropriate conditions in the mixture proportional reversed hazard rate models. This case can be viewed as a kind of anti-symmetry.

(15) Reversely closed but not closed under other appropriate conditions in the mixture proportional reversed hazard rate models. This case can be viewed as a kind of anti-symmetry.

(16) Closed but not reversely closed under some appropriate conditions in the coherent systems with dependent and identically distributed components. This case can be viewed as a kind of anti-symmetry.

(17) Reversely closed but not closed under other appropriate conditions in the coherent systems with dependent and identically distributed components. This case can be viewed as a kind of anti-symmetry.

Author Contributions: Methodology, L.Y., D.K. and H.W.; Resources, H.W.; Software, H.W. All authors contributed equally to the manuscript. All authors have read and agreed to the published version of the manuscript. 
Funding: This paper was supported by the scientific research and innovation team of "digital economy serving port economy research" of Zhejiang Wanli University (Grant No. 202036).

Institutional Review Board Statement: Not applicable.

Informed Consent Statement: Not applicable.

Data Availability Statement: Not applicable.

Conflicts of Interest: The authors declare no conflict of interest.

\section{References}

1. Zee, A. Fearful Symmetry: The Search for Beauty in Modern Physics; Princeton University Press: Princeton, NJ, USA, 2015.

2. Din, A.; Li, Y. Controlling heroin addiction via age-structured modeling. Adv. Differ. Equ. 2020, 2020, 521. [CrossRef]

3. Din, A.; Li, Y.; Yusuf, A. Delayed hepatitis B epidemic model with stochastic analysis. Chaos Solitons Fractals 2021, 146, 110839. [CrossRef]

4. Kosec, P.; Skec, S.; Miler, D. A comparison of the tolerance analysis methods in the open-loop assembly. Adv. Prod. Eng. Manag. 2020, 15, 44-56. [CrossRef]

5. Din, A.; Li, Y.; Khan, T.; Zaman, G. Mathematical analysis of spread and control of the novel corona virus (COVID-19) in China. Chaos Solitons Fractals 2020, 141, 110286. [CrossRef]

6. Petitjean, M. Chirality and symmetry measures: A transdisciplinary review. Entropy 2003, 5, 271-312. [CrossRef]

7. Tahmasebi, S.; Keshavarz, A.; Longobardi, M.; Mohammadi, R. A Shift-Dependent Measure of Extended Cumulative Entropy and Its Applications in Blind Image Quality Assessment. Symmetry 2020, 12, 316. [CrossRef]

8. Barlow, R.E. Statistical Inference under Order Restrictions; Wiley: New York, NY, USA, 1972.

9. Barlow, R.E.; Campo, R. Total Time on Test Processes and Applications to Failure Data Analysis. In Reliability and Fault Tree Analysis; Society for Industrial and Applied Mathematics: Berkeley, CA, USA, 1975.

10. Klefsjö, B. On aging properties and total time on test transforms. Scand. J. Stat. 1982, 37-41.

11. Bartoszewicz, J. Stochastic order relations and the total time on test transform. Stat. Probab. Lett. 1995, 22, 103-110. [CrossRef]

12. Bartoszewicz, J. Tail orderings and the total time on test transform. Appl. Math. 1996, 24, 77-86. [CrossRef]

13. Bartoszewicz, J. Applications of a general composition theorem to the star order of distributions. Stat. Probab. Lett. 1998, 38, 1-9. [CrossRef]

14. Bartoszewicz, J.; Benduch, M. Some properties of the generalized TTT transform. J. Stat. Plan. Inference 2009, 139, $2208-2217$. [CrossRef]

15. Pham, T.; Turkkan, N. The Lorenz and the scaled total-time-on-test transform curves: A unified approach. IEEE Trans. Reliab. 1994, 43, 76-84. [CrossRef]

16. Li, X.; Shaked, M. A general family of univariate stochastic orders. J. Stat. Plan. Inference 2007, 137, 3601-3610. [CrossRef]

17. Nanda, A.K.; Shaked, M. Partial ordering and aging properties of order statistics when the sample size is random: A brief review. Commun. Stat. Theory Methods 2008, 37, 1710-1720. [CrossRef]

18. Shaked, M.; Shanthikumar, J.G. Stochastic Orders; Springer: New York, NY, USA, 2007.

19. Franco-Pereira, A.M.; Shaked, M. The total time on test transform and the decreasing percentile residual life aging notion. Stat. Methodol. 2014, 18, 32-40. [CrossRef]

20. Kochar, S.C.; Li, X.; Shaked, M. The total time on test transform and the excess wealth stochastic orders of distributions. Adv. Appl. Probab. 2002, 34, 826-845. [CrossRef]

21. Jewitt, I. Choosing between risky prospects: The characterization of comparative statics results, and location independent risk. Manag. Sci. 1989, 35, 60-70. [CrossRef]

22. Fagiuoli, E.; Pellerey, F.; Shaked, M. A characterization of the dilation order and its applications. Stat. Pap. 1999, 40, 393. [CrossRef]

23. Nair, N.U.; Sankaran, P. Some new applications of the total time on test transforms. Stat. Methodol. 2013, 10, 93-102. [CrossRef]

24. Vineshkumar, B.; Nair, N.U.; Sankaran, P. Stochastic orders using quantile-based reliability functions. J. Korean Stat. Soc. 2015, 44, 221-231. [CrossRef]

25. Barlow, R.E.; Proschan, F. Statistical Theory of Reliability and Life Testing; Hold, Reinhart and Wiston , Inc.: Silver Spring, MD, USA, 1981.

26. Nair, N.U.; Sankaran, P.; Kumar, B.V. Total time on test transforms of order $\mathrm{n}$ and their implications in reliability analysis. J. Appl. Probab. 2008, 45, 1126-1139. [CrossRef]

27. Nanda, A.K.; Sankaran, P.; Sunoj, S. Renyi's residual entropy: A quantile approach. Stat. Probab. Lett. 2014, 85, 114-121. [CrossRef]

28. Sunoj, S.; Sankaran, P. Quantile based entropy function. Stat. Probab. Lett. 2012, 82, 1049-1053. [CrossRef]

29. Sunoj, S.; Sankaran, P.; Nanda, A.K. Quantile based entropy function in past lifetime. Stat. Probab. Lett. 2013, 83, 366-372. [CrossRef]

30. Li, X.; Shaked, M. The observed total time on test and the observed excess wealth. Stat. Probab. Lett. 2004, 68, 247-258. [CrossRef]

31. Shaked, M.; Shanthikumar, J.G.; Tong, Y. Stochastic orders and their applications. SIAM Rev. 1995, 37, 477-478. 
32. Marshall, A.W.; Olkin, I. A new method for adding a parameter to a family of distributions with application to the exponential and Weibull families. Biometrika 1997, 84, 641-652. [CrossRef]

33. Sankaran, P.; Jayakumar, K. On proportional odds models. Stat. Pap. 2008, 49, 779-789. [CrossRef]

34. Navarro, J.; del Aguila, Y.; Asadi, M. Some new results on the cumulative residual entropy. J. Stat. Plan. Inference 2010, 140, 310-322. [CrossRef]

35. Chandler, K. The distribution and frequency of record values. J. R. Stat. Soc. Ser. B Methodol. 1952, 14, 220-228. [CrossRef]

36. Khaledi, B.E.; Amiripour, F.; Hu, T.; Shojaei, S.R. Some new results on stochastic comparisons of record values. Commun. Stat. Theory Methods 2009, 38, 2056-2066. [CrossRef]

37. Kundu, C.; Nanda, A.K.; Hu, T. A note on reversed hazard rate of order statistics and record values. J. Stat. Plan. Inference 2009, 139, 1257-1265. [CrossRef]

38. Zhao, P.; Balakrishnan, N. Stochastic comparison and monotonicity of inactive record values. Stat. Probab. Lett. 2009, 79, 566-572. [CrossRef]

39. Zarezadeh, S.; Asadi, M. Results on residual Rényi entropy of order statistics and record values. Inf. Sci. 2010, 180, 4195-4206. [CrossRef]

40. Li, X.; Zhang, S. Some new results on Rényi entropy of residual life and inactivity time. Probab. Eng. Inf. Sci. 2011, 25, 237-250. [CrossRef]

41. Kang, D. Further results on closure properties of LPQE order. Stat. Methodol. 2016, 25, 23-35. [CrossRef]

42. Kang, D. Some new results on the LQE ordering. Stat. Methodol. 2016, 32, 218-235. [CrossRef]

43. Kang, D. Some results on DDCRE class of life distributions. Sankhya A 2015, 77, 351-363. [CrossRef]

44. Kang, D.; Yan, L. On the dynamic cumulative residual quantile entropy ordering. Stat. Methodol. 2016, 32, 14-35. [CrossRef]

45. Yan, L.; Kang, D. Some new results on the Rényi quantile entropy Ordering. Stat. Methodol. 2016, 33, 55-70. [CrossRef]

46. Ahsanullah, M. Record Statistics; Nova Science Publishers: New York, NY, USA, 1995.

47. Arnold, B.C.; Balakrishnan, N.; Nagaraja, H.N. Records; John Wiley \& Sons: New York, NY, USA, 1998.

48. Dziubdziela, W.; Kopociński, B. Limiting properties of the k-th record values. Appl. Math. 1976, 2, 187-190. [CrossRef]

49. Gupta, R.C.; Gupta, R.D. Proportional reversed hazard rate model and its applications. J. Stat. Plan. Inference 2007, 137, 3525-3536. [CrossRef]

50. Di Crescenzo, A.; Longobardi, M. On cumulative entropies. J. Stat. Plan. Inference 2009, 139, 4072-4087. [CrossRef]

51. Nanda, A.K.; Paul, P. Some results on generalized residual entropy. Inf. Sci. 2006, 176, 27-47. [CrossRef]

52. Abbasnejad, M.; Arghami, N.R.; Morgenthaler, S.; Borzadaran, G. On the dynamic survival entropy. Stat. Probab. Lett. 2010, 79, 1962-1971. [CrossRef]

53. Navarro, J.; del Águila, Y.; Sordo, M.A.; Suárez-Llorens, A. Stochastic ordering properties for systems with dependent identically distributed components. Appl. Stoch. Models Bus. Ind. 2013, 29, 264-278. [CrossRef] 